\title{
Numerical coupling of Landau damping and Raman amplification.
}

\author{
R. Belaouar ${ }^{1,2}$, T. Colin ${ }^{2}$, G. Gallice ${ }^{1}$ \\ 1 CEA CESTA, SIS, BP 233114 Le barp, France. \\ 2 IMB University Bordeaux 1, Inria Futurs project MC2 \\ 351 cours de la Libération, 33405 Talence, France
}

\begin{abstract}
In this paper, we present a numerical model for laser-plasma interaction involving Raman instability and Landau damping. This model exhibits three main difficulties. The first one is the coupling of PDE's posed both in Fourier space and in physical space. The second one is a three wave resonance condition that has to be verified. The third one is the boundary conditions. We overcome these difficulties using respectively a splitting scheme, a numerical dispersion relation and absorbing boundary conditions. We present some comparison between several phenomena that are involved and the influence of the Raman amplification and the Landau damping.
\end{abstract}

\section{Contents}

1 Introduction and physical context 1

2 The model and its properties. 3

2.1 The equations and their non-dimensional form. . . . . . . . . . . . . 3

2.2 Dimensionless system . . . . . . . . . . . . . . . . . 5

2.3 Some basic properties. . . . . . . . . . . . . . . . . 6

3 Numerical approximation. $\quad 7$

3.1 The numerical scheme. . . . . . . . . . . . . . . . . . . 7

$3.2 \quad L^{2}$ stability result . . . . . . . . . . . . . . . . . 12

3.3 The three wave resonance condition. . . . . . . . . . . . . . 15

3.3 .1 Numerical illustration . . . . . . . . . . . . . . . . . . 16

3.4 The boundary conditions. . . . . . . . . . . . . . . . 18

3.4.1 Boundary condition for the Schrödinger equation . . . . . . . . . 18

3.4.2 Boundary condition for the wave equation . . . . . . . . . . . . 22

3.4.3 The boundary conditions for the Zakharov system. . . . . . . . . . 27

3.4.4 Boundary condition for the full system . . . . . . . . . . . . . 28

4 Numerical results $\quad 29$

4.1 Basic simulations . . . . . . . . . . . . . . . . . . . . . . 29

4.2 Influence of the boundary conditions . . . . . . . . . . . . . . 35

4.2.1 Neumann boundary conditions for $A_{C}$ and $A_{R} \ldots \ldots . \ldots 36$

4.2.2 Neumann boundary conditions for $\delta n \ldots \ldots . \ldots . \ldots 37$

4.3 Influence of the dispersion relation . . . . . . . . . . . . . . . . . 39

4.4 Influence of the Landau damping process . . . . . . . . . . . . . . . . 40

4.5 Comparison with a first study . . . . . . . . . . . . . . . . . . 41 


\section{Introduction and physical context}

The interaction of an intense laser pulse with a plasma is a complex physical phenomenon. Numerical simulation plays a key role in its understanding. One of the main goal is to simulate nuclear fusion by inertial confinement in a laboratory. We therefore need some accurate and reliable numerical models of laser-plasma interactions. Vlasov or particle-incell (PIC) simulations have been used for a complete description of the problem. However, these kinetic simulations have difficulties in studying weak instabilities and long time behaviors because they need to resolve very small spatial and temporal scales. For the same reasons, it is not possible to use Euler-Maxwell equations.

Recently, M. Colin and T. Colin [5], starting from [16], derived a complete set of quasi-linear Zakharov equations describing the interactions between the laser fields, the stimulated Raman processes, the electronic plasma waves and the low-frequency variations of density of the ions. The system involves four Schrödinger equations coupled by quasilinear terms and a wave equation and describes a three-waves interaction. Physically, the lasers interacts with the plasma, part of it backscattered through a Raman-type process to create an electron plasma wave. These three waves interact in order to create a lowfrequency variation of density which has itself an influence on the three preceding waves. However, this model that is obtained starting from the fluid equations does not take into account the kinetic effects such the Landau damping effect which is a wave-particle process which occurs in under-dense plasma. The Landau damping process is especially important in the context of fusion by inertial confinement by lasers because electrons are accelerated to high energy and this induces a preheat of the fusion fuel and reduces the target gain. This wave-particle process corresponds to a resonant effect between the electrons of the plasma and the plasma electronic waves. This effect implies an exchange of energy between electrons and the plasma waves. As a result, the plasma waves are damped.

Of course many description of the Landau damping phenomenon exists in the literature starting at the kinetic level (see Glassey-Schaeffer [12], P. Degond [9] for example). Here we do not try to obtain such precise models. We try to couple a simpler one with the Raman process in order to be able to simulate the main feature of the Raman-Landau interaction.

In order to obtain a system describing this wave-particle process we complete the system used in [5] by using the model derived in [2]. The aim of this paper is to perform mathematically and numerically the coupling of these models that describes the interaction of the variation of the density of ions with the slowly varying envelope of the plasma electronic waves, the spatial mean value of the distribution function of the electrons, the laser field and the Raman component. We want to achieve two goals. The first one is to investigate what is the influence of the Landau damping process on the saturation of the Raman amplification. The second question we want to address is the influence of the Raman instability on the model [2] in terms of the number of accelerated electrons.

For that study, we use the scheme introduced in [5], a time-splitting discretization for the Landau damping term and a implicit finite difference scheme for the distribution function of the electrons. The main difficulties are the following :

i) First we have to couple the equations of the Raman model of [5] with those of the Landau model of [2]. This is done numerically by using a splitting strategy in section 3.1. The Landau damping model consist in two partial differential equations, one is 
posed in the physical space, the other one in Fourier space. The Fourier transform of some field occurs explicitly in the partial differential equations. The coupling of such models in the context of boundary value is not obvious especially because of the electronic plasma waves have to be considered in a periodic framework in the model [2].

ii) The second difficulty is the three-wave interaction condition. Indeed, it is shown in [5] that the Raman system that is obtained relies on an interaction condition. In our context, this condition means that the couple $\left(k_{1}, \omega_{1}\right)$ involved in the system is such that $e^{i\left(k_{1} x-\omega_{1} t\right)}$ is an exact solution to a linear Schrödinger equation. It is a phase matching condition. After discretization, one obtains a numerical phase matching condition that is different from that of the continuous case. In order to handle this difficulty, we define and use $\omega_{1 d}$, the frequency given by the numerical dispersion relation. This is done in section 3.2.

iii) The third difficulty is linked to the spatial box. For physical considerations, we can not use periodic boundary conditions since we want that once a pulse ( the laser part or the Raman part) hits the boundary, it does not interact anymore with the remaining part of the system. We therefore introduce some kind of absorbing boundary conditions. It is the object of section 3.3.

The outline of the paper is the following one. Section 2 is devoted to a complete presentation of the model and we introduced a dimensionless form. In section 3 , in order to solve the problem, we introduce an efficient numerical scheme and show some of its stability properties. Finally, in the last section, we will provide some numerical results in order to see how the coupling between Raman amplification and Landau damping process works.

\section{The model and its properties.}

\subsection{The equations and their non-dimensional form.}

In this section, we introduce the one dimensional system describing the Raman amplification and the Landau damping process. We consider here an homogeneous plasma where collisions between the particles (electrons and ions) and the gravitational field are neglected. We want to describe the interaction of a laser field with this plasma and the physical phenomenon quoted previously. We use the following model (see [5]) :

$$
\begin{aligned}
i\left(\partial_{t} A_{0}+\frac{k_{0} c^{2}}{\omega_{0}} \partial_{y} A_{0}\right)+ & \frac{c^{2}}{2 \omega_{0}}\left(1-\frac{k_{0}^{2} c^{2}}{\omega_{0}^{2}}\right) \partial_{y}^{2} A_{0}=\frac{\omega_{p e}^{2}}{2 n_{0} \omega_{0}} \delta n A_{0} \\
& -\frac{e}{2 m_{e} \omega_{0}}\left(\partial_{y} \cdot E\right) A_{R} e^{-i\left(k_{1} y-\omega_{1} t\right)}, \\
i\left(\partial_{t} A_{R}+\frac{k_{R} c^{2}}{\omega_{R}} \partial_{y} A_{R}\right)+ & \frac{c^{2}}{2 \omega_{R}}\left(1-\frac{k_{R}^{2} c^{2}}{\omega_{R}^{2}}\right) \partial_{y}^{2} A_{R}=\frac{\omega_{p e}^{2}}{2 n_{0} \omega_{R}} \delta n A_{R} \\
& -\frac{e}{2 m_{e} \omega_{R}}\left(\partial_{y} \cdot E^{*}\right) A_{0} e^{i\left(k_{1} y-\omega_{1} t\right)},
\end{aligned}
$$




$$
\begin{aligned}
& i\left(\partial_{t} E+\nu * E\right)+\frac{3 v_{t h}^{2}}{2 \omega_{p e}} \partial_{y}^{2} E=\frac{\omega_{p e}}{2 n_{0}} \delta n E \\
& +\frac{e \omega_{p e}}{2 c^{2} m_{e}} \partial_{y}\left(A_{R}^{*} A_{0} e^{i\left(k_{1} y-\omega_{1} t\right)}\right), \\
& \left(\partial_{t}^{2}-c_{s}^{2} \partial_{y}^{2}\right) \delta n=\frac{1}{4 \pi m_{i}} \partial_{y}^{2}\left(|E|^{2}+\frac{\omega_{p e}^{2}}{c^{2}}\left(\left|A_{0}\right|^{2}+\left|A_{R}\right|^{2}\right)\right), \\
& \widehat{\nu}(\xi, t)=-\frac{\pi \omega_{p e}^{3}}{2 \xi n_{0}|\xi| \xi} \partial_{v} F_{e}\left(\frac{\omega_{p e}}{\xi}\right), \\
& \partial_{t} F_{e}=\partial_{v}\left(D(v, t) \partial_{v} F_{e}\right), \quad D(v, t)=\frac{e^{2}}{2 m_{e}^{2}|v|}\left|\widehat{E}\left(\xi=\frac{\omega_{p e}}{v}, t\right)\right|^{2} .
\end{aligned}
$$

Here $A_{0}$ is the envelope of the vector potential of the incident electromagnetic laser field, $A_{R}$ is the envelope of the vector potential of Raman backscattered light, $E$ is the slowly varying amplitude of the high-frequency electronic plasma waves, $\delta n$ the low-frequency variation of the density of the ions, $F_{e}$ the spatially averaged electron distribution function, $\widehat{\nu}$ the spatial fourier transform of $\nu$ corresponding to the Landau damping rate and $u^{*}$ is the complex conjugate of $u$. In this work we consider that the laser propagates in the positive $y$ direction and we stay in the one dimensional framework.

This system involves three Schrödinger equations coupled by quasi-linear terms and the low frequency fluctuation of density given by the wave equation (2.4). The electron distribution function satisfies a heat equation where the diffusion coefficient $D(v, t)$ depends on the density spectral energy of electron plasma waves. The constants are defined by:

- $c$ is the speed of light in the vacuum, $e$ is the elementary electric charge,

- $m_{e}$ and $m_{i}$ are respectively the electron's and ion's mass,

- $n_{0}$ is the mean background density of the plasma,

- $T_{e}$ is the electronic temperature,

- $\omega_{p e}, v_{t h e}$ and $c_{s}$ are respectively the electronic plasma pulsation, the thermal velocity of electrons and the acoustic velocity of ions given by

$$
\omega_{p e}=\sqrt{\frac{4 \pi e^{2} n_{0}}{m_{e}}}, v_{\text {the }}=\sqrt{\frac{T_{e}}{m_{e}}}, c_{s}=\sqrt{\frac{T_{e}}{m_{i}}},
$$

- $\omega_{0}, \omega_{R}, \omega_{p e}+\omega_{1}$ are respectively the laser pump frequency, the Raman component frequency and the electronic plasma wave frequency,

- $k_{0}, k_{R}, k_{1}$ are respectively the laser pump wave number, the Raman component wave number and the electronic plasma wave number. 
Note that $\left(\omega_{0}, \omega_{R}, \omega_{1}\right)$ and $\left(k_{0}, k_{R}, k_{1}\right)$ have to satisfy the three-waves resonance condition

$$
\begin{aligned}
& \omega_{0}=\omega_{R}+\omega_{p e}+\omega_{1}, \\
& k_{0}=k_{R}+k_{1},
\end{aligned}
$$

in order to have an efficient process. Here $\left(k_{0}, \omega_{0}\right),\left(k_{R}, \omega_{R}\right)$ correspond to electromagnetic waves while, $\left(k_{1}, \omega_{p e}+\omega_{1}\right)$ corresponds to electronic plasma waves and the dispersion relations are therefore

$$
\begin{aligned}
& \omega_{0}^{2}=\omega_{p e}^{2}+c^{2} k_{0}^{2}, \\
& \omega_{R}^{2}=\omega_{p e}^{2}+c^{2} k_{R}^{2}, \\
& \left(\omega_{p e}+\omega_{1}\right)^{2}=\omega_{p e}^{2}+3 v_{\text {the }}^{2} k_{1}^{2} .
\end{aligned}
$$

Note that, the last relation can be written approximatively $\omega_{1} \approx \frac{3 v_{t h e}^{2} k_{1}^{2}}{2 \omega_{p e}}$.

The full electric field can then be recovered as follows

$$
E_{f}(t, x)=i \frac{\omega_{0}}{c_{0}} A_{0} e^{i\left(k_{0} y-\omega_{0} t\right)}+i \frac{\omega_{R}}{c} A_{R} e^{i\left(k_{R} y-\omega_{R} t\right)}+E e^{-i \omega_{p e} t}+c . c .
$$

where c.c. denotes the complex conjugate.

With this model, we can recover the model used in [5] by taking $\nu=0$ in (2.3) to obtain system (2.1)-(2.4) which was derived from a bi-fluid Euler-Maxwell system. We can also recover the system used in [2] by fixing the potentials $A_{R}$ and $A_{0}$ to obtain system (2.3)(2.6) where in (2.3), we have a fixed source term given by $\partial_{y}\left(A_{R}^{*} A_{0} e^{i\left(k_{1} y-\omega_{1} t\right)}\right)$. Concerning the wave-particle process, the model is valid for bounded velocity that are also bounded away from zero (see [8]). Let $\Omega_{v}$ be the velocity domain on which equation (2.6) has to be satisfied and $\Omega_{\xi}=\left\{\xi \in \mathbb{R}\right.$ s.t. $\left.\exists v \in \Omega_{v}, v=\frac{1}{\xi}\right\}$. The domain $\Omega_{\xi}$ will therefore be taken under the form, $\Omega_{\xi}=[-A,-a] \cup[a, A]$ with $0<a<A$ (see section 2). Note the term $\widehat{\nu}$ is only defined on $\Omega_{\xi}$ by (2.5) and is extended by 0 outside the domain $\Omega_{\xi}$.

\subsection{Dimensionless system}

We now introduce a dimensionless form of (2.1)-(2.6).

We use $T=\frac{1}{\omega_{0}}$ as time scale and $L=\frac{1}{k_{0}}$ as space scale and introduce

$$
\begin{aligned}
& \widetilde{A_{0}}=\frac{e}{m_{e} c^{2}} A_{0}, \quad \widetilde{A_{R}}=\frac{e}{m_{e} c^{2}} A_{R}, \\
& \widetilde{E}=\frac{e}{m_{e} v_{e} \omega_{p e}} E, \quad \widetilde{\widehat{\nu}}=\frac{1}{\omega_{p e}} \widehat{\nu}, \\
& \widetilde{k_{1}}=\frac{k_{1}}{k_{0}}, \quad \widetilde{\omega_{1}}=\frac{\omega_{1}}{\omega_{0}}, \\
& \widetilde{F_{e}}=\frac{v_{\text {the }}}{n_{0}} F_{e}, \quad \widetilde{\delta n}=\frac{1}{n_{0}} \delta n .
\end{aligned}
$$

Omitting the tildes, we get the following system :

$$
\begin{aligned}
i\left(\partial_{t} A_{0}+v_{0} \partial_{y} A_{0}\right)+ & \frac{1}{2} v_{0}\left(1-v_{0}\right) \partial_{y}^{2} A_{0}=\frac{\omega_{p e}^{2}}{2 \omega_{0}^{2}} \delta n A_{0} \\
& -\frac{k_{0}}{k_{D e}} \frac{\omega_{p e}^{2}}{\omega_{0}^{2}}\left(\partial_{y} E\right) A_{R} e^{-i\left(k_{1} y-\omega_{1} t\right)}
\end{aligned}
$$




$$
\begin{gathered}
i\left(\partial_{t} A_{R}+v_{R} \partial_{y} A_{R}\right)+\frac{\omega_{0}}{2 \omega_{R}}\left(1-\frac{k_{R}^{2} c^{2}}{\omega_{R}^{2}}\right) \partial_{y}^{2} A_{R}=\frac{\omega_{p e}^{2}}{2 \omega_{R} \omega_{0}} \delta n A_{R} \\
-\frac{k_{0}}{k_{D e}} \frac{\omega_{p e}^{2}}{\omega_{0} \omega_{R}}\left(\partial_{y} E^{*}\right) A_{0} e^{i\left(k_{1} y-\omega_{1} t\right)} \\
i\left(\partial_{t} E+\frac{\omega_{p e}}{\omega_{0}} \nu * E\right)+\frac{3 k_{0}^{2}}{2 k_{D e}^{2}} \frac{\omega_{p e}}{\omega_{0}} \partial_{y}^{2} E=\frac{\omega_{p e}}{2 \omega_{0}} \delta n E \\
\quad+\frac{c^{2} k_{0} k_{D e}}{4 \omega_{0} \omega_{p e}} \partial_{y}\left(A_{R}^{*} A_{0} e^{i\left(k_{1} y-\omega_{1} t\right)}\right) \\
\left(\partial_{t}^{2}-v_{s}^{2} \partial_{y}^{2}\right) \delta n=\frac{m_{e}}{4 m_{i}} \frac{k_{0}^{2}}{k_{D e}^{2}} \frac{\omega_{p e}^{2}}{\omega_{0}^{2}} \partial_{y}^{2}|E|^{2}+\frac{m_{e}}{4 m_{i}} v_{s} \partial_{y}^{2}\left(\left|A_{0}\right|^{2}+\left|A_{R}\right|^{2}\right) \\
\widehat{\nu}(t, \xi)=-\frac{\pi k_{D e}^{2}}{2 k_{0}^{2} \xi|\xi|} \partial_{v} F_{e}\left(v=\frac{k_{D e}}{k_{0} \xi}\right), \\
\partial_{t} F_{e}=\partial_{v}\left(D(v, t) \partial_{v} F_{e}\right), \quad D(v, t)=\frac{k_{D e} \omega_{p e}}{k_{0} \omega_{0}|v|}\left|\widehat{E}\left(\xi=\frac{k_{D e}}{k_{0} v}, t\right)\right|^{2}
\end{gathered}
$$

where $v_{0}=\frac{c^{2} k_{0}^{2}}{\omega_{0}^{2}}$ is the group velocity of $A_{0}, v_{R}=\frac{c^{2} k_{0} k_{R}}{\omega_{0} \omega_{R}}$ is the group velocity of $A_{R}$, $v_{s}=\frac{c_{s}^{2} k_{0}^{2}}{\omega_{0}^{2}}$ is the velocity of the ion acoustic waves and $k_{D e}=\frac{1}{\lambda_{D e}}$ where $\lambda_{D e}$ denotes the Debye length. In order to study the quasi-linear diffusion equation (2.17), it is more convenient to use the variable $\xi=\frac{k_{D e}}{k_{0} v}$ instead of $v$. Then denoting

$$
H(t, \xi)=F_{e}\left(t, \frac{k_{D e}}{k_{0} \xi}\right)
$$

the distribution function, (2.17) becomes

$$
\partial_{t} H-\frac{\omega_{p e} k_{0}^{2}}{\omega_{0} k_{D e}^{2}} \xi^{2} \partial_{\xi}\left(|\xi|^{3}|\hat{E}(t, \xi)|^{2} \partial_{\xi} H\right)=0,
$$

and the Landau damping rate reads

$$
\widehat{\nu}(t, \xi)=\operatorname{sgn}(\xi) \frac{\pi}{2} \frac{k_{D e}^{3}}{k_{0}^{3}} \partial_{\xi} H_{e}(t, \xi) .
$$

\subsection{Some basic properties.}

One first have an energy conservation given by

Proposition 2.1. For any regular solution of $(2.12)-(2.17)$, one has

$$
\frac{d}{d t} \int\left(2 \frac{\omega_{0}}{\omega_{p e}}\left|A_{0}\right|^{2}+\frac{\omega_{R}}{\omega_{p e}}\left|A_{R}\right|^{2}+\frac{4 v_{t h e}^{2}}{c^{2}}|E|^{2}\right) d y+\frac{4 k_{D e} v_{t h e}^{2}}{k_{0} c^{2}} \int \widehat{\nu}(t, \xi)|\widehat{E}|^{2}(t, \xi) d \xi=0 .
$$


Proof : Multiply $(2.12)$ by $2 A_{0}^{*},(2.13)$ by $A_{R}^{*},(2.14)$ by $E^{*}$, integrating over $\mathbb{R}$, summing the results, taking the imaginary part, using the Plancherel formula for the Landau damping term and denoting by $\theta=k_{1} x-\omega_{1} t$, give

$$
\begin{aligned}
& \frac{1}{2} \partial_{t} \int\left(2 \frac{\omega_{0}}{\omega_{p e}}\left|A_{0}\right|^{2}+\frac{\omega_{R}}{\omega_{p e}}\left|A_{R}\right|^{2}+\frac{4 v_{t h e}^{2}}{c^{2}}|E|^{2}\right) d y+\frac{4 k_{D e} v_{t h e}^{2}}{k_{0} c^{2}} \int \widehat{\nu}(t, \xi)|\widehat{E}|^{2}(t, \xi) d \xi \\
& =\Im \int\left(-2 \partial_{y} E A_{R} A_{0}^{*} e^{-i \theta}-\partial_{y} E^{*} A_{R}^{*} A_{0} e^{i \theta}+\partial_{y}\left(A_{R}^{*} A_{0} e^{i \theta}\right) E^{*}\right) d y, \\
& =\Im \int\left(2 \partial_{y} E^{*} A_{R}^{*} A_{0} e^{i \theta}-\partial_{y} E^{*} A_{R}^{*} A_{0} e^{i \theta}-A_{R}^{*} A_{0} \partial_{y} E^{*} e^{i \theta}\right) d y, \\
& =0 . \quad
\end{aligned}
$$

Moreover, one has some maximum principle on (2.16)-(2.17) that show that the convolution term in (2.14) is indeed a damping term. More precisely, we recall the following result imported from [2] :

Proposition 2.2. If $\hat{\nu}(0, \xi) \geq 0$ for all $\xi$, then one have

$$
\hat{\nu}(t, \xi)=\operatorname{sgn}(\xi) \partial_{\xi} H(t, \xi) \geq 0,
$$

for all $\xi \in \mathbb{R}$ and $t>0$.

Remark 2.1. If the the initial distribution function of electron is a Maxwellian then one has $\hat{\nu}(0, \xi) \geq 0$. Proposition (2.1) expresses the decay of the electromagnetic energy due to the Landau damping process between the electron plasma waves and electrons.

\section{Numerical approximation.}

In this section, we present an numerical scheme for system (2.12)-(2.17) endowed with the following initial conditions

$$
\begin{array}{ll}
A_{0}(0, y)=A_{00}(y), & A_{R}(0, y)=A_{R 0}(y), \quad E(0, y)=E_{0}(y), \\
\delta n(0, y)=\delta n_{0}(y), & \partial_{t} \delta n(0, y)=\delta n_{1}(y), \quad H(0, \xi)=H_{0}(\xi) .
\end{array}
$$

We work on the spatial domain $[0, L]$ and we use a regular mesh in space. The spatial mesh size is $h=\delta y$ with $h=L / N$ for $N=2 M$ being an even number, the time step being $\delta t>0$ and let the grid points and the time step be

$$
y_{j}=j h, t_{k}=k \delta t, j=0,1, \ldots, N, k=0,1,2 \ldots
$$

with $y_{0}=0$ and $y_{N}=L$. We also define the sequence $\left(\xi_{j}=\frac{2 \pi j}{L}\right)_{j=-\frac{N}{2}, \ldots, 0, \ldots, \frac{N}{2}-1}$ as the regular mesh grid for the frequencies with $\delta \xi=\frac{2 \pi}{L}$. Furthermore, let $A_{0 j}^{k}, A_{R j}^{k}, E_{j}^{k}$, $\delta n_{j}^{k}$, and $\widehat{\nu}_{j}^{k}$ be the approximations of $A_{0}\left(t_{k}, x_{j}\right), A_{R}\left(t_{k}, x_{j}\right), E\left(t_{k}, x_{j}\right), \delta n\left(t_{k}, x_{j}\right), \widehat{\nu}\left(t_{k}, \xi_{j}\right)$. Then, in order to be consistent with the evaluation of $\widehat{\nu}\left(t_{k}, \xi_{j}\right)=\operatorname{sgn}\left(\xi_{j}\right) \partial_{\xi} H\left(t_{k}, \xi_{j}\right)$, we approximate $H$ on the grid $\left(\xi_{j+\frac{1}{2}}\right)_{j}$ defined by $\xi_{j+\frac{1}{2}}=\frac{2 \pi(j+1 / 2)}{L}$.

The numerical scheme used in [16] for the wave part of our model is a pseudo-spectral 
discretization. The authors observed some aliasing errors due to the nonlinear and quasilinear terms and they were obliged to truncate the Fourier transform of the different fields. In [6], a fractional-step, Crank-Nicholson-type scheme with relaxation directly inspired by that of C. Besse for NLS (see [4]) is proposed for the quasi-linear system. For the acoustic part, they used an energy-preserving finite difference scheme introduced by Glassey (see [11]).

We present our scheme in three parts. In the first part, we give the scheme in itself in the case of periodic boundary condition (section 3.1). In the second part, we deal with the three-waves resonance conditions and we explain how we overcome this difficulty (section 3.3). In the third part, we explain how one can construct some kind of transparent boundary conditions for our problem (section 3.4).

\subsection{The numerical scheme.}

The Landau damping rate in the Schrödinger equation evolving the electronic plasma waves is given in Fourier space and it is given by a diffusion equation while the Zakharov part of the model is posed in the physical space. Therefore we have to use a spectral method to evaluate this convolution operator. In the other hand we can not use a spectral method for the linear part of (2.12)-(2.13) since we deal with transport operators. To overcome this difficulty, we introduce a splitting technique on the Landau damping process in order to separate the Raman amplification process and the Landau damping. Therefore, as we will see in numerical applications, since the group velocity of the electronic plasma waves is closed to zero, this allow us to use a spectral method (periodic boundary conditions for $E$ ) to solve the Landau damping part. Finally, for the Raman amplification, we use the numerical scheme introduced in [6]. We now describe more precisely the different step of our method. If at time $t^{k}$, we know $A_{0 j}^{k}, A_{R j}^{k}, E_{j}^{k}, \delta n_{j}^{k}, H_{j+\frac{1}{2}}^{k}$ and $\widehat{\nu}_{j}^{k}$, we construct $A_{0 j}^{k+1}, A_{R j}^{k+1}, E_{j}^{k+1}, \delta n_{j}^{k+1}, H_{j+\frac{1}{2}}^{k+1}$ and $\widehat{\nu}_{j}^{k+1}$ in three steps.

In a first step, we use a scheme for the quasi-linear diffusion equation. In a second one, we introduce the scheme used in [6] for (2.12)-(2.14) without the convolution operator describing the Landau process and finally, using a fast Fourier transform, we compute the modification given by the Landau damping rate on the electronic plasma waves.

\section{Step 1 : The diffusion.}

In order to evaluate the approximation of $H\left(t_{k}, \xi_{j+\frac{1}{2}}\right)$, we use an implicit difference scheme for the diffusion equation :

$$
\partial_{t} H-\xi^{2} \partial_{\xi}\left(|\xi|^{3}|\widehat{E}|^{2} \partial_{\xi} H\right)=0, \quad \xi \in \Omega
$$

where $\Omega=\left[-\xi_{2},-\xi_{1}\right] \cup\left[\xi_{1}, \xi_{2}\right],\left(\xi_{2}>\xi_{1}>0\right)$, with $\xi_{1}=\frac{2 \pi\left(j_{1}+1 / 2\right)}{L}>\frac{2 \pi}{L}, \xi_{2}=\frac{2 \pi\left(j_{2}+1 / 2\right)}{L}<$ $\frac{2 \pi(M-1)}{L}$.

The boundary conditions are

$$
\begin{aligned}
& \partial_{\xi} H\left(., \pm \xi_{1}\right)=0, \\
& H\left(.,-\xi_{2}\right)=H\left(0,-\xi_{2}\right), \\
& H\left(., \xi_{2}\right)=H\left(0, \xi_{2}\right) .
\end{aligned}
$$


The finite difference scheme reads :

$$
\frac{1}{\delta t}\left(H_{j+\frac{1}{2}}^{k+1}-H_{j+\frac{1}{2}}^{k}\right)+(A H)_{j+\frac{1}{2}}^{k+1}=0
$$

where $(A H)_{j+\frac{1}{2}}^{k+1}$ is a discretization of $-\xi^{2} \partial_{\xi}\left(\beta(t, \xi) \partial_{\xi} H\right)$ in a conservative form at the point $\xi_{j+\frac{1}{2}}$ and time $t_{k+1}$. We choose $A$ such that:

$$
(A H)_{j+\frac{1}{2}}^{k}=-\frac{\xi_{j+\frac{1}{2}}^{2}}{\xi_{j+1}-\xi_{j}}\left[\beta_{j+1}^{k-1} \frac{H_{j+\frac{3}{2}}^{k}-H_{j+\frac{1}{2}}^{k}}{\xi_{j+\frac{3}{2}}-\xi_{j+\frac{1}{2}}}-\beta_{j}^{k-1} \frac{H_{j+\frac{1}{2}}^{k}-H_{j-\frac{1}{2}}^{k}}{\xi_{j+\frac{1}{2}}-\xi_{j-\frac{1}{2}}}\right],
$$

where $\beta_{j}^{k}$ is the approximation of $\beta(t, \xi)=|\xi|^{3}|\widehat{E}|^{2}$. Then, with this scheme, we can evaluate $\widehat{\nu}\left(\xi_{j}, t^{k+1}\right)$ on the frequency grid with the following centered finite difference scheme :

$$
\widehat{\nu}_{j}^{k+1}=\operatorname{sgn}\left(\xi_{j}\right) \frac{H_{j+\frac{1}{2}}^{k+1}-H_{j-\frac{1}{2}}^{k+1}}{\xi_{j+\frac{1}{2}}-\xi_{j-\frac{1}{2}}} .
$$

As shown in [2], one take

$$
\beta_{j}^{k}=\frac{\xi_{j+\frac{1}{2}}^{2} \xi_{j-\frac{1}{2}}^{2}}{\left|\xi_{j+\frac{1}{2}}+\xi_{j-\frac{1}{2}}\right|}\left|\hat{E}_{j}^{k}\right|^{2}
$$

in order to ensure the following conservation relation

$$
\sum_{j} \frac{1}{\xi_{j+\frac{1}{2}}^{4}} H_{j+\frac{1}{2}}^{k+1}=\sum_{j} \frac{1}{\xi_{j+\frac{1}{2}}^{4}} H_{j+\frac{1}{2}}^{k}+\delta t \sum_{j} \widehat{\nu}_{j}^{k+1}\left|\hat{E}_{j}^{k}\right|^{2}
$$

which corresponds to the energy exchange between electrons and the electronics plasma waves. One also have a numerical maximum principle for $\widehat{\nu}$. If $\widehat{\nu}_{0}$ satisfies

$$
\widehat{\nu}_{0}\left(\xi_{j}\right) \geq 0, \quad j=-\frac{N}{2}, \ldots, \frac{N}{2}-1,
$$

then for all $k>0$

$$
\widehat{\nu}_{j}^{k} \geq 0, \quad j=-\frac{N}{2}, \ldots, \frac{N}{2}-1 .
$$

In order to illustrate how the quasi-linear diffusion works on the solution, we have computed the diffusion equation with a diffusion coefficient given by a fixed electric field

$$
E(t, x)=e^{i\left(k_{1} x-\omega_{1} t\right)} e^{-\frac{\left(x-\frac{L}{2}\right)^{2}}{2 \triangle L^{2}}},
$$

with $L=2000, \triangle L=50$ and $k_{1}=0.45$.

The initial electron distribution function is assumed to be a Maxwellian,

$$
F_{e 0}(v)=\frac{1}{\sqrt{2 \pi}} \exp \left(-\frac{v^{2}}{2}\right)
$$

which gives the following initial condition for the Landau damping rate

$$
\widehat{\nu}(0, \xi)=\sqrt{\frac{\pi}{8}} \frac{1}{|\xi|^{3}} e^{-\frac{1}{2 \xi^{2}}} .
$$



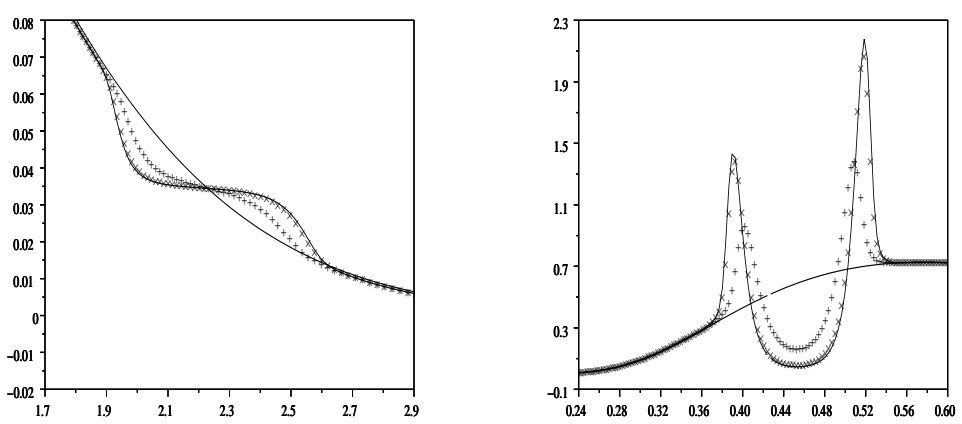

Figure 1: The left plot corresponds with the electron distribution function $F_{e}$ at different time in function of the velocity $v$ and the right plot corresponds with the Landau damping rate at different time in function of the frequency $\xi$.

We can remark on figure 1 that the electron distribution function is flattened near the phase velocity $v_{\phi}=\frac{1}{k_{1}}=2.22$ and since the Landau damping rate depends on the slope of the electron distribution, we can see that $\widehat{\nu}$ tends toward zero near $\xi=k_{1}$. We will see in section 4 , what happens when we take into account the time evolution of the diffusion coefficient.

\section{Step 2 : The Raman amplification.}

For the Raman amplification, we introduce a fractional-step, Crank-Nicolson-type scheme with relaxation introduced by of C. Besse for NLS equation (see [4]). For the acoustic part, we use the scheme introduced by Glassey (see [11]). We consider centered discretization for each spatial differential operator. Therefore, $\partial_{y}$ is approximated by the centered finite difference operator $D_{0}$ :

$$
\left(D_{0} E\right)_{i}=\frac{E_{i+1}-E_{i-1}}{2 \delta y}
$$

and $\partial_{y}^{2}$ by $D_{+} D_{-}$:

$$
\left(D_{+} D_{-} E\right)_{i}=\frac{E_{i+1}-2 E_{i}+E_{i-1}}{\delta y^{2}} .
$$

For this first step of the splitting, the scheme reads:

$$
\begin{aligned}
& i \frac{A_{0}^{k+1}-A_{0}^{k}}{\delta t}+\left(i v_{0} D_{0}+\alpha_{0} D_{+} D_{-}\right)\left(\frac{A_{0}^{k+1}+A_{0}^{k}}{2}\right)=\beta_{0}\left(\frac{\delta n^{k+1}+\delta n^{k}}{2}\right)\left(\frac{A_{0}^{k+1}+A_{0}^{k}}{2}\right) \\
& -\frac{\gamma_{0}}{2} \phi^{k+\frac{1}{2}}\left(\frac{A_{R}^{k+1}+A_{R}^{k}}{2}\right) e^{-i \theta^{k+\frac{1}{2}}}-\frac{\gamma_{0}}{2} \psi^{k+\frac{1}{2}}\left(\frac{D_{0} E^{k+1}+D_{0} E^{k}}{2}\right) e^{-i \theta^{k+\frac{1}{2}}}
\end{aligned}
$$




$$
\begin{gathered}
i \frac{A_{R}^{k+1}-A_{R}^{k}}{\delta t}+\left(i v_{R} D_{0}+\alpha_{R} D_{+} D_{-}\right)\left(\frac{A_{R}^{k+1}+A_{R}^{k}}{2}\right)=\beta_{R}\left(\frac{\delta n^{k+1}+\delta n^{k}}{2}\right)\left(\frac{A_{R}^{k+1}+A_{R}^{k}}{2}\right) \\
-\gamma_{R}\left(\phi^{k+\frac{1}{2}}\right)^{*}\left(\frac{A_{0}^{k+1}+A_{0}^{k}}{2}\right) e^{i \theta^{k+\frac{1}{2}}}, \\
i \frac{E^{k+1}-E^{k}}{\delta t}+\alpha_{E} D_{+} D_{-}\left(\frac{E^{k+1}+E^{k}}{2}\right)=\beta_{E}\left(\frac{\delta n^{k+1}+\delta n^{k}}{2}\right)\left(\frac{E^{k+1}+E^{k}}{2}\right) \\
+\gamma_{E} D_{0}\left[\left(\psi^{k+\frac{1}{2}}\right)^{*}\left(\frac{A_{0}^{k+1}+A_{0}^{k}}{2}\right) e^{i \theta^{k+\frac{1}{2}}}\right], \\
\frac{\delta n^{k+1}-2 \delta n^{k}+\delta n^{k-1}}{\delta t^{2}}-v_{s}^{2} D_{+} D_{-}\left(\frac{\delta n^{k+1}+\delta n^{k-1}}{2}\right)=\delta_{1} D_{+} D_{-}\left(\left|E^{k}\right|^{2}\right)+ \\
\delta_{2} D_{+} D_{-}\left(\left|A_{0}^{k}\right|^{2}+\left|A_{R}^{k}\right|^{2}\right)
\end{gathered}
$$

for the time step of length $\delta t$.

The constants $\left(\alpha_{0}, \beta_{0}, \gamma_{0}\right),\left(\alpha_{R}, \beta_{R}, \gamma_{R}\right),\left(\alpha_{E}, \beta_{E}, \gamma_{E}\right),\left(\delta_{1}, \delta_{2}\right)$, are the following one

$$
\begin{aligned}
& \alpha_{0}=\frac{1}{2} v_{0}\left(1-v_{0}\right), \beta_{0}=\frac{\omega_{p e}^{2}}{2 \omega_{0}^{2}}, \gamma_{0}=\frac{k_{0} \omega_{p e}^{2}}{k_{D e} \omega_{0}^{2}}, \\
& \alpha_{R}=\frac{\omega_{0}}{2 \omega_{R}}\left(1-\frac{c^{2} k_{R}^{2}}{\omega_{R}^{2}}\right), \beta_{R}=\frac{\omega_{p e}^{2}}{2 \omega_{0} \omega_{R}}, \gamma_{R}=\frac{k_{0} \omega_{p e}^{2}}{k_{D e} \omega_{0} \omega_{R}}, \\
& \alpha_{E}=\frac{3 k_{0}^{2} \omega_{p e}}{2 k_{D e}^{2} \omega_{0}}, \beta_{E}=\frac{\omega_{p e}}{2 \omega_{0}}, \gamma_{E}=\frac{c^{2} k_{0} k_{D e}}{4 \omega_{0} \omega_{p e}}, \\
& \delta_{1}=\frac{m_{e}}{m_{i}} \frac{k_{0}^{2}}{k_{D e}^{2}} \beta_{E}^{2}, \delta_{2}=\frac{m_{e}}{4 m_{i}} v_{s} .
\end{aligned}
$$

The auxiliary functions $\phi$ and $\psi$ are given by

$$
\frac{\phi^{k+\frac{1}{2}}+\phi^{k-\frac{1}{2}}}{2}=D_{0} E^{k}, \frac{\psi^{k+\frac{1}{2}}+\psi^{k-\frac{1}{2}}}{2}=A_{R}^{k} .
$$

$\phi^{k+\frac{1}{2}}$ and $\psi^{k+\frac{1}{2}}$ are prediction respectively of $\partial_{y} E$ and $A_{R}$ at time $\left(k+\frac{1}{2}\right) \delta t$. Moreover the value $\phi^{-\frac{1}{2}}$ and $\psi^{-\frac{1}{2}}$ are obtained by explicit integration of the system on one half-time step backward.

Therefore the discretization of the phase $\theta^{k+\frac{1}{2}}$ is given by

$$
\theta^{k+\frac{1}{2}}=k_{1} y-\omega_{1}\left(k+\frac{1}{2}\right) \delta t .
$$

We now denote by $\widetilde{A_{0}}, \widetilde{A_{R}}, \widetilde{E}, \widetilde{\delta n}$ the solutions of (3.8)-(3.11). 


\section{Step 3 : The Landau damping.}

In the following step, we have to solve

$$
\begin{array}{rlrl}
\partial_{t} A_{0} & =0, & A_{0}(0, y) & =\widetilde{A_{0}}(y), \\
\partial_{t} A_{R} & =0, \quad A_{R}(0, y)=\widetilde{A_{R}}(y), \\
\partial_{t} E+\nu * E & =0, \quad E(0, y)=\widetilde{E}(y),
\end{array}
$$

which corresponds to the modification of the electronic plasma waves due to the Landau process. Using the Fourier transform for (3.15), one gets exactly

$$
\begin{array}{r}
A_{0}\left(t^{k+1}, y_{j}\right)=\widetilde{A_{0}}\left(y_{j}\right), \\
A_{R}\left(t^{k+1}, y_{j}\right)=\widetilde{A_{R}}\left(y_{j}\right), \\
\widehat{E}\left(t^{k+1}, \xi_{j}\right)=\widehat{\widetilde{E}}\left(\xi_{j}\right) \exp \left(-\int_{t^{k}}^{t^{k+1}} \widehat{\nu}\left(s, \xi_{j}\right) d s\right)
\end{array}
$$

and using a trapezoidal rule, we obtain

$$
\widehat{E}\left(t^{k+1}, \xi_{j}\right) \approx \widehat{\widetilde{E}}\left(\xi_{j}\right) \exp \left(-\frac{\delta t}{2}\left(\widehat{\nu}_{j}^{k+1}+\widehat{\nu}_{j}^{k}\right)\right) .
$$

Using the inverse discrete Fourier transform, we get

$$
E_{j}^{k+1}=\sum_{l=-M}^{M-1} \widehat{E}_{l}^{k+1} e^{i \xi_{l} x_{j}}, \quad j=1, . ., N .
$$

\section{2 $\quad L^{2}$ stability result}

The first stability result concerns the semi-discretization in time of the model. This semidiscretization in time is obtained by discretization of (2.12)-(2.17) only in time and using the same time-splitting as described before. In order not to be confuse, in this part, we will denote by $u, r, w, \mu, f$ the semi-discretized version of the physical unknowns $A_{0}, A_{R}$, $E, \nu, \delta n$. Let $u^{0}, r^{0}, w^{0}, \mu^{0}$ and $f^{0}$ be the initial conditions of $u, r, w, \mu$ and $f$. The semi discretization scheme reads

- Step 1 : The diffusion

In the first step, we solve the semi-discretization in time of the quasi-linear diffusion evolving the electron distribution function on the interval $\left[t_{k}, t_{k+1}\right]$. By using the usual implicit Euler scheme in time, the first step reads

$$
\begin{aligned}
& \frac{H^{k+1}-H^{k}}{\delta t}-\xi^{2} \partial_{\xi}\left(\beta\left(t^{k}, \xi\right) \partial_{\xi} H^{k+1}\right)=0, \quad \xi \in \Omega_{\xi} \\
& \mu^{k+1}(\xi)=\operatorname{sgn}(\xi) \partial_{\xi} H^{k+1} .
\end{aligned}
$$

Note that $\mu^{k+1}(\xi)$ is extended by zero outside the domain $\Omega_{\xi}$.

- Step 2 : The ion density fluctuation 
The semi-discretization in time of the wave equation reads

$$
\frac{f^{k+1}-2 f^{k}+f^{k-1}}{\delta t^{2}}-v_{s}^{2} \partial_{y}^{2}\left(\frac{f^{k+1}+f^{k-1}}{2}\right)=\delta_{1} \partial_{y}^{2}\left|w^{k}\right|^{2}+\delta_{2} \partial_{y}^{2}\left(\left|u^{k}\right|^{2}+\left|r^{k}\right|^{2}\right) .
$$

- Step 3 : The three wave interaction

Here, we find $(\widetilde{u}, \widetilde{r}, \widetilde{w})$ in terms of $\left(u^{k}, r^{k}, w^{k}\right)$ by solving

$$
\begin{aligned}
& i \frac{\widetilde{u}-u^{k}}{\delta t}+\left(i v_{C} \partial_{y}+\alpha_{0} \partial_{y}^{2}\right)\left(\frac{\widetilde{u}+u^{k}}{2}\right)=\beta_{0}\left(\frac{f^{k+1}+f^{k}}{2}\right)\left(\frac{\widetilde{u}+u^{k}}{2}\right) \\
& -\frac{\gamma_{0}}{2} \phi^{k+\frac{1}{2}}\left(\frac{\widetilde{r}+r^{k}}{2}\right) e^{-i \theta^{k+\frac{1}{2}}}-\frac{\gamma_{0}}{2} \psi^{k+\frac{1}{2}}\left(\frac{\partial_{y} \widetilde{w}+\partial_{y} w^{k}}{2}\right) e^{-i \theta^{k+\frac{1}{2}}}, \\
& i \frac{\widetilde{r}-r^{k}}{\delta t}+\left(i v_{R} \partial_{y}+\alpha_{R} \partial_{y}^{2}\right)\left(\frac{\widetilde{r}+r^{k}}{2}\right)=\beta_{R}\left(\frac{f^{k+1}+f^{k}}{2}\right)\left(\frac{\widetilde{r}+r^{k}}{2}\right) \\
& -\gamma_{R}\left(\phi^{k+\frac{1}{2}}\right)^{*}\left(\frac{\widetilde{u}+u^{k}}{2}\right) e^{i \theta^{k+\frac{1}{2}}}, \\
& i \frac{\widetilde{w}-w^{k}}{\delta t}+\alpha_{E} \partial_{y}^{2}\left(\frac{\widetilde{w}+w^{k}}{2}\right)=\beta_{E}\left(\frac{f^{k+1}+f^{k}}{2}\right)\left(\frac{\widetilde{w}+w^{k}}{2}\right) \\
& +\gamma_{E} \partial_{y}\left[\left(\psi^{k+\frac{1}{2}}\right)^{*}\left(\frac{\widetilde{u}+u^{k}}{2}\right) e^{i \theta^{k+\frac{1}{2}}}\right],
\end{aligned}
$$

where the auxiliary functions $\phi$ and $\psi$ are defined by

$$
\frac{\phi^{k+\frac{1}{2}}+\phi^{k-\frac{1}{2}}}{2}=\partial_{y} w^{k}, \quad \frac{\psi^{k+\frac{1}{2}}+\psi^{k-\frac{1}{2}}}{2}=r^{k}
$$

- Step 4 : The Landau damping

Finally, in the last step, in order to compute the contribution of the Landau damping process, we solve

$$
\begin{aligned}
& \partial_{t} u=0, u\left(t^{k}, y\right)=\widetilde{u}(y), \\
& \partial_{t} r=0, r\left(t^{k}, y\right)=\widetilde{r}(y), \\
& \partial_{t} w+\mu * w=0, w\left(t^{k}, y\right)=\widetilde{w}(y),
\end{aligned}
$$

which give us

$$
\begin{aligned}
u\left(t^{k+1}, y\right) & =\widetilde{u}(y), \\
r\left(t^{k+1}, y\right) & =\widetilde{r}(y), \\
\widehat{w}\left(t^{k+1}, \xi\right) & =\widehat{\widetilde{w}}(\xi) \exp \left(-\int_{t_{k}}^{t_{k+1}} \widehat{\mu}(s, \xi) d s\right)
\end{aligned}
$$

and we recover $w\left(t^{k+1}, y\right)$ by taking the inverse Fourier transform of (3.33) and using a trapezoidal rule. 
Proposition 3.1. ( $L^{2}$ stability) If the initial data $\mu^{0}$ satisfy $\widehat{\mu^{0}}(\xi) \geq 0$ for all $\xi$, then for all $k>0$, any solution $\left(u^{k}, r^{k}, w^{k}\right)$ given by the semi-discrete scheme (3.21)-(3.33) satisfies

$$
\int_{\mathbb{R}} 2 \alpha_{1}\left|u^{k}\right|^{2}+\alpha_{2}\left|r^{k}\right|^{2}+\alpha_{3}\left|w^{k}\right|^{2} d y \leq \int_{\mathbb{R}} 2 \alpha_{1}\left|u^{0}\right|^{2}+\alpha_{2}\left|r^{0}\right|^{2}+\alpha_{3}\left|w^{0}\right|^{2} d y
$$

where $\alpha_{1}=\frac{\omega_{0}}{\omega_{p e}}, \alpha_{2}=\frac{\omega_{R}}{\omega_{p e}}$ and $\alpha_{3}=4 \frac{v_{\text {the }}^{2}}{c^{2}}$.

Proof : Using (3.31), (3.32) and the Parseval formula for $w^{k+1}$, we have

$$
\begin{aligned}
\int_{\mathbb{R}} 2 \alpha_{1}\left|u^{k+1}\right|^{2}+\alpha_{2}\left|r^{k+1}\right|^{2}+\alpha_{3}\left|w^{k+1}\right|^{2} d y= & \int_{\mathbb{R}} 2 \alpha_{1}|\widetilde{u}|^{2}+\alpha_{2}|\widetilde{r}|^{2} d y \\
& +\alpha_{3} \int_{\mathbb{R}}|\widehat{\widetilde{w}}|^{2} \exp \left(-\delta t\left(\widehat{\mu}^{k}(\xi)+\widehat{\mu}^{k+1}(\xi)\right)\right) d \xi .
\end{aligned}
$$

In order to conclude, we need the following version of the maximum principle.

Lemma 3.1. If $\mu^{0}(\xi) \geq 0$ for all $\xi$, then for all $k>0$, any solution $\mu^{k}$ of $(3.21)-(3.22)$ satisfy $\mu^{k}(\xi) \geq 0$ for all $\xi$.

Proof : see $[2]$.

Using the lemma 3.1 and again the Parseval formula, one gets

$$
\int_{\mathbb{R}} 2 \alpha_{1}\left|u^{k+1}\right|^{2}+\alpha_{2}\left|r^{k+1}\right|^{2}+\alpha_{3}\left|w^{k+1}\right|^{2} d y \leq \int_{\mathbb{R}} 2 \alpha_{1}|\widetilde{u}|^{2}+\alpha_{2}|\widetilde{r}|^{2}+\alpha_{3}|\widetilde{w}|^{2} d y .
$$

In order to conclude, we need to prove that

$$
\int_{\mathbb{R}} 2 \alpha_{1}|\widetilde{u}|^{2}+\alpha_{2}|\widetilde{r}|^{2}+\alpha_{3}|\widetilde{w}|^{2} d y=\int_{\mathbb{R}} 2 \alpha_{1}\left|u^{k}\right|^{2}+\alpha_{2}\left|r^{k}\right|^{2}+\alpha_{3}\left|w^{k}\right|^{2} d y .
$$

To this aim, we compute

$$
2 \alpha_{1} \int_{\mathbb{R}}(3.24)\left(\frac{\widetilde{u}+u^{k}}{2}\right)^{*}+\alpha_{2} \int_{\mathbb{R}}(3.25)\left(\frac{\widetilde{r}+r^{k}}{2}\right)^{*}+\alpha_{3} \int_{\mathbb{R}}(3.26)\left(\frac{\widetilde{w}+w^{k}}{2}\right)^{*},
$$

and take the imaginary part. This yields

$$
\begin{aligned}
& \frac{1}{2 \delta t} \int_{\mathbb{R}}\left(2 \alpha_{1}|\widetilde{u}|^{2}+\alpha_{2}|\widetilde{r}|^{2}+\alpha_{3}|\widetilde{w}|^{2}\right)-\frac{1}{2 \delta t} \int_{\mathbb{R}}\left(2 \alpha_{1}\left|u^{k}\right|^{2}+\alpha_{2}\left|r^{k}\right|^{2}+\alpha_{3}\left|w^{k}\right|^{2}\right) \\
& =-\operatorname{Im} \int_{\mathbb{R}} \phi^{k+\frac{1}{2}}\left(\frac{\widetilde{r}+r^{k}}{2}\right) e^{-i \theta^{k+\frac{1}{2}}}\left(\frac{\widetilde{u}+u^{k}}{2}\right)^{*} \\
& -\operatorname{Im} \int_{\mathbb{R}} \psi^{k+\frac{1}{2}}\left(\frac{\partial_{y} \widetilde{w}+\partial_{y} w^{k}}{2}\right) e^{-i \theta^{k+\frac{1}{2}}}\left(\frac{\widetilde{u}+u^{k}}{2}\right)^{*} \\
& -\operatorname{Im} \int_{\mathbb{R}}\left(\phi^{k+\frac{1}{2}}\right)^{*}\left(\frac{\widetilde{u}+u^{k}}{2}\right) e^{i \theta^{k+\frac{1}{2}}}\left(\frac{\widetilde{r}+r^{k}}{2}\right)^{*} \\
& +\operatorname{Im} \int_{\mathbb{R}} \partial_{y}\left(\left(\psi^{k+\frac{1}{2}}\right)^{*}\left(\frac{\widetilde{u}+u^{k}}{2}\right) e^{i \theta^{k+\frac{1}{2}}}\right)\left(\frac{\widetilde{w}+w^{k}}{2}\right)^{*} \\
& =-\mathrm{I}-\mathrm{II}-\mathrm{III}+\mathrm{IV} .
\end{aligned}
$$


It is clear that $\mathrm{I}=-$ III. Moreover

$$
\mathrm{IV}=-\operatorname{Im} \int_{\mathbb{R}}\left(\psi^{k+\frac{1}{2}}\right)^{*}\left(\frac{\widetilde{u}+u^{k}}{2}\right) e^{i \theta^{k+\frac{1}{2}}} \partial_{y}\left(\frac{\widetilde{w}+w^{k}}{2}\right)^{*}=\mathrm{II} .
$$

The result follows since

$$
\int_{\mathbb{R}} 2 \alpha_{1}|\widetilde{u}|^{2}+\alpha_{2}|\widetilde{r}|^{2}+\alpha_{3}|\widetilde{w}|^{2}=\int_{\mathbb{R}} 2 \alpha_{1}\left|u^{k}\right|^{2}+\alpha_{2}\left|r^{k}\right|^{2}+\alpha_{3}\left|w^{k}\right|^{2}
$$

Remark 3.1. Proposition 3.1 holds for the full discretization of the system in a periodic framework. This can be shown by using the fact that if we assume $\hat{\mu}(t, \xi)=0$ for all $(t, \xi)$, then it is shown in [6] that any solution of (3.24)-(3.26) satisfies

$$
2 \alpha_{1}\left|u^{k}\right|_{2}^{2}+\alpha_{2}\left|r^{k}\right|_{2}^{2}+\alpha_{3}\left|w^{k}\right|_{2}^{2}=2 \alpha_{1}\left|u^{0}\right|_{2}^{2}+\alpha_{2}\left|r^{0}\right|_{2}^{2}+\alpha_{3}\left|w^{0}\right|_{2}^{2}
$$

where

$$
|f|_{2}^{2}=\sum_{j=1}^{N}\left|f_{j}\right|^{2}
$$

is the $l^{2}$ discrete norm. In our case, we have to include the Landau damping term $\hat{\mu}(t, \xi)$. The key point is that we have used a time splitting discretization which allows us to write

$$
2 \alpha_{1}\left|u^{k+1}\right|_{2}^{2}+\alpha_{2}\left|r^{k+1}\right|_{2}^{2}+\alpha_{3}\left|w^{k+1}\right|_{2}^{2}=2 \alpha_{1}|\widetilde{u}|_{2}^{2}+\alpha_{2}|\widetilde{r}|_{2}^{2}+\alpha_{3} \sum_{j=1}^{N}\left|w_{j}^{k+1}\right|^{2},
$$

where $w_{j}^{k+1}$ is defined by (3.20). By using twice the Parseval formula, the maximum principle (3.7) and (3.19), we obtain that

$$
\sum_{j=1}^{N}\left|w_{j}^{k+1}\right|^{2} \leq \sum_{j=1}^{N}\left|\widetilde{w}_{j}\right|^{2}
$$

and the result follows :

$$
2 \alpha_{1}\left|u^{k+1}\right|_{2}^{2}+\alpha_{2}\left|r^{k+1}\right|_{2}^{2}+\alpha_{3}\left|w^{k+1}\right|_{2}^{2} \leq 2 \alpha_{1}\left|u^{k}\right|_{2}^{2}+\alpha_{2}\left|r^{k}\right|_{2}^{2}+\alpha_{3}\left|w^{k}\right|_{2}^{2}
$$

\subsection{The three wave resonance condition.}

As noted in the introduction, we expect an exponential growth on $A_{R}$ which corresponds to the decomposition of the laser $A_{0}$ into a backscattered electromagnetic wave $A_{R}$ and an electronic plasma waves $E$. The classical matching conditions for this three waves resonance interactions is

$$
k_{0}=k_{R}+k_{1}, \quad \omega_{0}=\omega_{R}+\omega_{p e}+\omega_{1},
$$

where

$$
\omega_{0}^{2}=\omega_{p e}^{2}+k_{0}^{2} c^{2}, \quad \omega_{R}^{2}=\omega_{p e}^{2}+k_{R}^{2} c^{2}, \quad\left(\omega_{p e}+\omega_{1}\right)^{2}=\omega_{p e}^{2}+3 v_{\text {the }}^{2} k_{1}^{2} .
$$


As recalled in the introduction and since $\omega_{1} \ll \omega_{p e}$ (see [5]), this last condition implies $\omega_{1} \approx \frac{3}{2} \omega_{p e}\left(k_{1} \lambda_{D e}\right)^{2}$ where $\lambda_{D e}=\frac{v_{t h e}}{\omega_{p e}}$ is the Debye's length.

This means that $\left(k_{1}, \omega_{1}\right)$ satisfies the dispersion relation of the linear part of $(2.14)$ and therefore the term $A_{R}^{*} A_{0} e^{i\left(k_{1} y-\omega_{1} t\right)}$ is resonant and the process will be efficient. More precisely, the equation on $E$ can be written in (neglecting $\delta n$ and considering $A_{R}$ and $A_{0}$ as given)

$$
i \partial_{t} E+\frac{\lambda_{D}^{2} \omega_{p e}}{2} \partial_{y}^{2} E=A e^{i\left(k_{1} y-\omega_{1} t\right)} .
$$

Let $L$ be a characteristic scale in space, $T$ in time. The dimensionless form of (3.39) reads:

$$
i \partial_{t} E+\frac{\lambda_{D}^{2} \omega_{p e} T}{2 L^{2}} \partial_{y}^{2} E=A T e^{i\left(\left(k_{1} L\right) y-\left(\omega_{1} T\right) t\right)} .
$$

Introduce $k_{1} L=\frac{k}{\varepsilon}, \omega_{1} T=\frac{\omega}{\varepsilon}$, and replace $A T$ by $A$. Since $\omega_{1}=\frac{3}{2} \omega_{p e} \lambda_{D}^{2} k_{1}^{2}$ and $\omega_{1} T=\frac{\omega}{\varepsilon}$ (with $\omega, k=O(1)$ ), one gets

$$
\omega_{p e} \frac{\lambda_{D}^{2} T}{L^{2}}=\frac{\omega_{1} T}{k_{1}^{2} L^{2}}=\frac{\varepsilon^{2} \omega}{k^{2} \varepsilon}=\frac{\omega}{k^{2}} \varepsilon=O(\varepsilon) .
$$

The dimensionless form of (3.39) is therefore

$$
i \partial_{t} E+\frac{\varepsilon \omega}{k^{2}} \partial_{y}^{2} E=A e^{i \frac{(k y-\omega t)}{\varepsilon}} .
$$

A standard WKB expansion shows that

$$
E=B e^{i \frac{(k y-\omega t)}{\varepsilon}}+O(\varepsilon),
$$

with

$$
i\left(\partial_{t} B+\frac{\omega}{k} \partial_{y} B\right)=A
$$

This implies a linear growth in time for $B$ that travels at the group velocity $\frac{\omega}{k}$. On the other hand, if the equation is

$$
i \partial_{t} E+\frac{\varepsilon \omega}{k^{2}} \partial_{y}^{2} E=A e^{i \frac{\left(k y-\omega^{\prime} t\right)}{\varepsilon}},
$$

with $\omega^{\prime} \neq \omega$, then $E$ will stay of size $O(\varepsilon)$. Of course, nonlinear versions of these results can be proved.

For numerical consideration, we can not take $\omega_{1}=\frac{3}{2} \omega_{p e}\left(k_{1} \lambda_{D e}\right)^{2}$. In fact, if we consider the following scheme

$$
i \frac{u_{j}^{k+1}-u_{j}^{k}}{\delta t}+\alpha D_{+} D_{-}\left(\frac{u^{k+1}+u^{k}}{2}\right)_{j}=\beta e^{i\left(k_{1} x_{j}-w_{1} t^{k+\frac{1}{2}}\right)}
$$

then the source term on the r.h.s of (3.41) will be not resonant with the discretization of the linear Schrödinger equations and the growth of the plasma electronic waves will be not efficient numerically. To overcome this difficulty, we take the frequency $\omega_{1}$ given by the numerical dispersion relation of the Crank-Nicholson scheme

$$
i \frac{u_{j}^{k+1}-u_{j}^{k}}{\delta t}+\alpha D_{+} D_{-}\left(\frac{u^{k+1}+u^{k}}{2}\right)_{j}=0 .
$$


By seeking the solution $\left(u_{j}^{k}\right)$ of (3.42) under the form $u_{j}^{k}=\gamma e^{i\left(k_{1} x_{j}-w_{1} t^{k+\frac{1}{2}}\right)}$ with $\gamma \neq 0$, we find that the discrete pulsation $w_{1 d}:=\omega_{1}$ has to satisfy the following dispersion relation

$$
\omega_{1 d}=\frac{2}{\delta t} \arctan \left(\alpha \delta t \frac{1-\cos \left(k_{1} \delta y\right)}{\delta y^{2}}\right)
$$

which is not the same relation than for the continuous linear Schrödinger equation $\omega_{1}=$ $\alpha k_{1}^{2}$. Nevertheless, when $\delta t \rightarrow 0$ and $\delta y \rightarrow 0$, one finds the continuous dispersion relation, since

$$
\omega_{1 d}=\alpha k_{1}^{2}-\frac{1}{12}\left(\alpha^{3} k^{6} \delta t^{2}+\alpha k^{4} \delta y^{2}\right)+\frac{1}{48} \alpha^{3} k^{8} \delta y^{2} \delta t^{2}+O\left(\delta y^{3}\right)+O\left(\delta t^{3}\right) .
$$

However, for finite values of $\delta t$ and $\delta y,(3.43)$ gives much better results than the continuous version, see below.

\subsubsection{Numerical illustration}

Here, in order to illustrate numerically this phase matching condition, we solve the following equation

$$
\begin{aligned}
& i \partial_{t} u+\alpha \partial_{y}^{2} u=\beta e^{i\left(k_{1} y-w_{1} t\right)}, y \in[0, L], \\
& u(0, y)=u_{0}(y), y \in[0, L], \\
& u(t, 0)=u(t, L) .
\end{aligned}
$$

by using the scheme (3.41) and we compare the results obtained with the theoretical disper-

sion relation $\omega_{1}=\alpha k_{1}^{2}$ and the numerical dispersion relation $\omega_{1}:=\omega_{1 d}=\frac{2}{\delta t} \arctan \left(\alpha \delta t \frac{1-\cos \left(k_{1} \delta y\right)}{\delta y^{2}}\right)$.

We take $u_{0}=0, \alpha=2, \beta=1, L=4 \pi, k_{1}=1$. Note that in this case, the exact solution of $(3.45)-(3.47)$ is

$$
u(t, y)= \begin{cases}t e^{i\left(k_{1} y-\omega_{1} t\right)} & \text { if } \omega_{1}=2 k_{1}^{2}, \\ \frac{1}{\omega_{1}-\alpha k_{1}^{2}} e^{i\left(k_{1} y-\omega_{1} t\right)} & \text { if } \omega_{1} \neq 2 k_{1}^{2} .\end{cases}
$$

In figure 2 , the maximum amplitude of the solution $u$ is plotted with $\delta t=\delta y=0.01$. We can see that when we use the numerical dispersion relation, the growth of the amplitude of the solution is an accordance with the growth of the exact solution. In this case, when we use the theoretical dispersion relation, the growth of amplitude of $u$ stops near the time $t=200$, that means that the source term $e^{i\left(k_{1} y-w_{1} t\right)}$ becomes non-resonant for large time for the Crank-Nicholson scheme. 


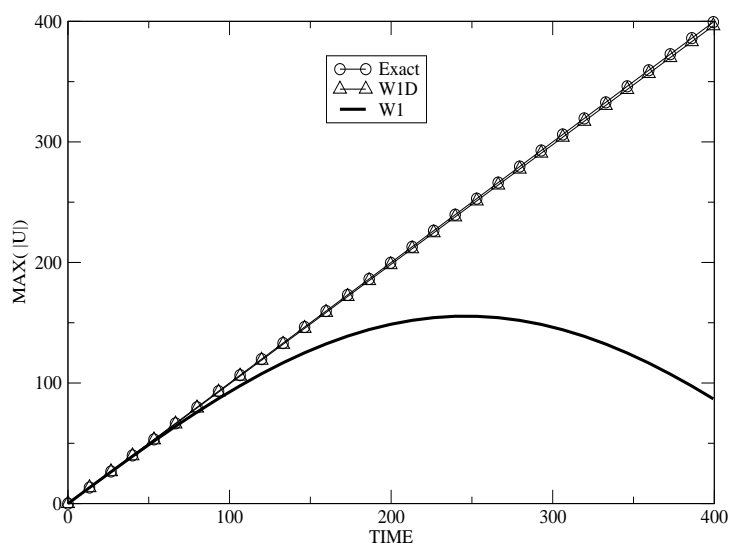

Figure 2: The maximum of amplitude of $|u|$ in function of time. The circle-line correspond to the exact solution, the triangle-line correspond to the discrete dispersion relation and the solid-line with the continuous dispersion relation for $\delta t=\delta y=0.01$. The triangle and circle lines are superposed.

In figure 3 , in order to see the influence of the time step, we take $\delta t=\delta y^{2}=0.0001$. In this case the numerical dispersion relation is closer to the theoretical dispersion relation than in the case where $\delta t=\delta y$. In fact, the plot corresponding with the theoretical dispersion relation shows us that the growth of the amplitude stops later than in the first case $(\delta t=\delta y)$. Anyway, on large time-scale, the results are different. 


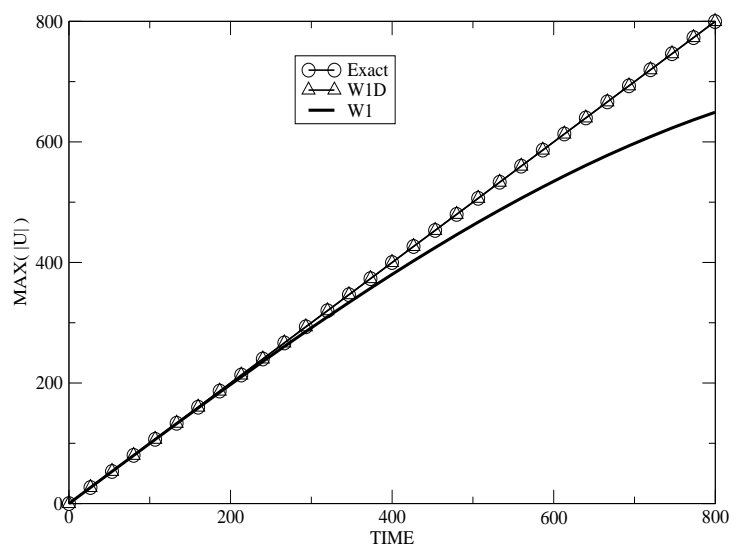

Figure 3: The maximum of amplitude of $|u|$ in function of time. The circle-line correspond to the exact solution, the triangle-line correspond to the discrete dispersion relation and the solid-line with the continuous dispersion relation for $\delta t=\delta y^{2}=0.0001$. The triangle and circle lines are superposed.

We will see in the numerical results for the full system that this choice of $\omega_{1 d}$ will be important in order to create electronic plasma waves that will exchange energy with electrons.

\subsection{The boundary conditions.}

For physical considerations, we use absorbing boundary conditions for $A_{0}$ and $A_{R}$ and $\delta n$. A lot of work involving transparent boundary conditions are available (see for example Di Menza [10], X. Antoine-C. Besse [1]). Here we take into account the particular physical setting and we use a very simple version of absorbing boundary conditions. In fact, in order to model a realistic plasma slab, non-periodic boundary conditions in (3.8)-(3.9) are imposed. This condition will ensure that if the Raman backscattered field, the laser field and the density fluctuation leave the simulation box no reflection can influence the propagation of the laser field and the growth of the Raman field. It appears physically that it is very important to impose absorbing boundary conditions for $A_{0}$ and $A_{R}$ and $\delta n$. In order to explain our choice of boundaries conditions, we introduce two simple independent (one for the Raman and laser fields and one for the density fluctuation) models on which one can explain our strategy.

\subsubsection{Boundary condition for the Schrödinger equation}

For the first model, we focus on the equations evolving the laser potential and the Raman backscattered potential :

$$
i\left(\partial_{t} A_{0}+v_{0} \partial_{y} A_{0}\right)+\alpha_{0} \partial_{y}^{2} A_{0}=\frac{\omega_{p e}^{2}}{2 \omega_{0}^{2}} \delta n A_{0}-\frac{k_{0}}{k_{D e}} \frac{\omega_{p e}^{2}}{\omega_{0}^{2}}\left(\partial_{y} E\right) A_{R} e^{-i\left(k_{1} y-\omega_{1} t\right)},
$$




$$
i\left(\partial_{t} A_{R}+v_{R} \partial_{y} A_{R}\right)+\alpha_{R} \partial_{y}^{2} A_{R}=\frac{\omega_{p e}^{2}}{2 \omega_{R} \omega_{0}} \delta n A_{R}-\frac{k_{0}}{k_{D e}} \frac{\omega_{p e}^{2}}{\omega_{0} \omega_{R}}\left(\partial_{y} E^{*}\right) A_{0} e^{i\left(k_{1} y-\omega_{1} t\right)}
$$

where $\alpha_{0}=\frac{1}{2} v_{0}\left(1-v_{0}\right)$ and $\alpha_{R}=\frac{\omega_{0}}{2 \omega_{R}}\left(1-\frac{c^{2} k_{R}^{2}}{\omega_{R}^{2}}\right)$. The key point is that in physical applications, $\left|v_{0}\right|$ and $\left|v_{R}\right|$ are close and the dispersion coefficient $\alpha_{0}, \alpha_{R}$ are closed to zero $\left(\alpha_{0, R} \approx 10^{-3}\right)$. It follows that the linear part of equations (3.48)-(3.49) is a dispersive perturbation of a linear transport equation. (see [5]-[6]). Therefore we will focus with the following Schrödinger equation

$$
\begin{aligned}
& i\left(\partial_{t} u+\partial_{y} u\right)+\varepsilon \partial_{y}^{2} u=0,0 \leq y \leq 1, \\
& u(t, 0)=0, \\
& u(0, y)=u_{0}(y),
\end{aligned}
$$

where $\varepsilon$ a small positive parameter. The goal of this study is to give an efficient absorbing boundary condition for (3.50)-(3.52) at point $y=1$. Since we deal with dispersive perturbation of a transport equation, the natural choice is to impose that the solution satisfies the transport equation at point $y=1$,

$$
\left(\partial_{t}+\partial_{y}\right) u(t, 1)=0 .
$$

It is not clear if this boundary condition is an absorbing boundary condition. The following proposition ensure this.

Proposition 3.2. Any solutions u of (3.50)-(3.53) satisfy

$$
\frac{d}{d t} \int\left|\partial_{y} u\right|^{2} d y=-\left(\left|\partial_{y} u(t, 0)\right|^{2}+\left|\partial_{y} u(t, 1)\right|^{2}\right) \text {. }
$$

Proof: By multiplying the equation (3.50) by $\partial_{t} u^{*}$, integrating in space, taking the real part, and integrating by part the dispersive term, we get

$$
\Re \int i \partial_{y} u \partial_{t} u^{*} d y-\frac{\varepsilon}{2} \frac{d}{d t} \int\left|\partial_{y} u\right|^{2} d y+\varepsilon \Re\left(\partial_{y} u(t, 1) \partial_{t} u^{*}(t, 1)\right)=0
$$

since $\partial_{t} u(t, 0)=0$. Now using the boundary condition (3.53), and plugging $i \partial_{t} u^{*}=$ $-i \partial_{y} u^{*}+\varepsilon \partial_{y}^{2} u^{*}$ in the first term of (3.54), we get

$$
\varepsilon \Re \int \partial_{y} u \partial_{y}^{2} u^{*}-\frac{\varepsilon}{2} \frac{d}{d t} \int\left|\partial_{y} u\right|^{2} d y-\varepsilon\left|\partial_{y} u(t, 1)\right|^{2}=0 .
$$

and the result follows since $\varepsilon \Re \int \partial_{y} u \partial_{y}^{2} u^{*}=\frac{\varepsilon}{2}\left|\partial_{y} u(t, 1)\right|^{2}-\frac{\varepsilon}{2}\left|\partial_{y} u(t, 0)\right|^{2}$.

Remark 3.2. Using Poincare's lemma, proposition 3.2 expresses the fact that with the boundary condition (3.53), the energy ( $L^{2}$-norm) decreases with time. Therefore, the estimate (3.54) show us that we can define the boundary trace of $\partial_{y} u$ at points $y=0$ and $y=1$.

For numerical illustration of this proposition, we use a Crank-Nicholson type scheme for (3.50)-(3.53). With the notations previously used, the scheme reads

$$
i \frac{u_{j}^{k+1}-u_{j}^{k}}{\delta t}+\left(i D_{0}+\varepsilon D_{+} D_{-}\right)\left(\frac{u^{k+1}+u^{k}}{2}\right)_{j}=0, \text { for } 2 \leq j \leq N-1 \text {. }
$$


We discretize the boundary condition (3.53) by using the following discretization of the linear transport equation

$$
\frac{u_{N}^{k+1}-u_{N}^{k}}{\delta t}+D_{+}\left(\frac{u^{k+1}+u^{k}}{2}\right)_{N}=0 .
$$

We have a discret version of proposition 3.2

Proposition 3.3. Any solutions $u^{k}$ of (3.57)-(3.58) satisfies

$$
\frac{\delta y}{\delta t}\left(\sum_{j=2}^{N-1}\left|\frac{u_{j+1}^{k+1}-u_{j}^{k+1}}{\delta y}\right|^{2}-\sum_{j=2}^{N-1}\left|\frac{u_{j+1}^{k}-u_{j}^{k}}{\delta y}\right|^{2}\right)=-\left(\left|\frac{\widetilde{u}_{2}-\widetilde{u}_{1}}{\delta y}\right|^{2}+\left|\frac{\widetilde{u}_{N}-\widetilde{u}_{N-1}}{\delta y}\right|^{2}\right)
$$

where $\widetilde{u}_{j}=\frac{u_{j}^{k+1}+u_{j}^{k}}{2}$.

Proof: The proof of this proposition follows the same line than that of the continuous case. We perform it by multiplying $(3.57)$ by $\left(u^{k+1}-u^{k}\right)^{*}$, we take the real part of the result and get

$$
\Re \sum_{j=2}^{N-1}\left(\frac{\widetilde{u}_{j+1}-\widetilde{u}_{j-1}}{2 \delta y}\right) i\left(u_{j}^{k+1}-u_{j}^{k}\right)^{*}+\Re \sum_{j=2}^{N-1}\left(\frac{\widetilde{u}_{j+1}-2 \widetilde{u}_{j}+\widetilde{u}_{j-1}}{\delta y^{2}}\right)\left(u_{j}^{k+1}-u_{j}^{k}\right)^{*}=0
$$

which is the sum of two terms $(I+I I=0)$. In order to compute the first term $I$, we substitute

$$
i\left(u_{j}^{k+1}-u_{j}^{k}\right)^{*}=-i \delta t \frac{\widetilde{u}_{j+1}^{*}-\widetilde{u}_{j-1}^{*}}{2 \delta y}+\varepsilon \delta t \frac{\widetilde{u}_{j+1}^{*}-2 \widetilde{u}_{j}^{*}+\widetilde{u}_{j-1}^{*}}{\delta y^{2}}
$$

in $I$ as in the continuous case. This gives

$$
\begin{aligned}
I & =\frac{\delta t}{2 \delta y^{3}} \Re \sum_{j=2}^{N-1}\left(\widetilde{u}_{j+1}-\widetilde{u}_{j-1}\right)\left(\widetilde{u}_{j+1}^{*}-2 \widetilde{u}_{j}^{*}+\widetilde{u}_{j-1}^{*}\right), \\
& =\frac{\delta t}{2 \delta y^{3}} \sum_{j=2}^{N-1}\left|\widetilde{u}_{j+1}\right|^{2}-\left|\widetilde{u}_{j-1}\right|^{2}-\frac{\delta t}{\delta y^{3}} \Re \sum_{j=2}^{N-1} \widetilde{u}_{j}^{*}\left(\widetilde{u}_{j+1}-\widetilde{u}_{j-1}\right), \\
& =\frac{\delta t}{2 \delta y^{3}}\left(\left|\widetilde{u}_{N}\right|^{2}+\left|\widetilde{u}_{N-1}\right|^{2}-\left(\left|\widetilde{u}_{1}\right|^{2}+\left|\widetilde{u}_{2}\right|^{2}\right)-2 \Re\left(\widetilde{u}_{N} \widetilde{u}_{N-1}^{*}\right)+2 \Re\left(\widetilde{u}_{1} \widetilde{u}_{2}^{*}\right)\right), \\
& =\frac{\delta t}{2 \delta y}\left(\left|\frac{\widetilde{u}_{N}-\widetilde{u}_{N-1}}{\delta y}\right|^{2}-\left|\frac{\widetilde{u}_{2}-\widetilde{u}_{1}}{\delta y}\right|^{2}\right) .
\end{aligned}
$$

Note that, the contribution of $I$ is the same that in the continuous case. The second term 
reads

$$
\begin{aligned}
I I= & \frac{1}{\delta y^{2}} \Re \sum_{j=2}^{N-1}\left(\widetilde{u}_{j+1}-2 \widetilde{u}_{j}+\widetilde{u}_{j-1}\right)\left(u_{j}^{k+1}-u_{j}^{k}\right)^{*} \\
I I= & \frac{1}{\delta y^{2}} \Re \sum_{j=2}^{N-1}\left(\widetilde{u}_{j+1}-\widetilde{u}_{j}\right)\left(u_{j}^{k+1}-u_{j}^{k}\right)^{*}-\frac{1}{\delta y^{2}} \Re \sum_{j=2}^{N-1}\left(\widetilde{u}_{j+1}-\widetilde{u}_{j}\right)\left(u_{j+1}^{k+1}-u_{j+1}^{k}\right)_{3 .}^{*} . \\
I I= & \frac{1}{\delta y^{2}} \Re \sum_{j=2}^{N-1}\left(\widetilde{u}_{j+1}-\widetilde{u}_{j}\right)\left(\left(u_{j}^{k+1}-u_{j}^{k}\right)^{*}-\left(u_{j+1}^{k+1}-u_{j+1}^{k}\right)^{*}\right) \\
& +\frac{1}{\delta y^{2}} \Re\left(\widetilde{u}_{N}-\widetilde{u}_{N-1}\right)\left(u_{N}^{k+1}-u_{N}^{k}\right)^{*}, \\
I I= & -\frac{1}{2} \sum_{j=2}^{N-1}\left(\left|\frac{u_{j+1}^{k+1}-u_{j}^{k+1}}{\delta y}\right|^{2}-\sum_{j=2}^{N-1}\left|\frac{u_{j+1}^{k}-u_{j}^{k}}{\delta y}\right|^{2}\right) \\
& +\frac{1}{\delta y^{2}} \Re\left(\widetilde{u}_{N}-\widetilde{u}_{N-1}\right)\left(u_{N}^{k+1}-u_{N}^{k}\right)^{*} .
\end{aligned}
$$

Now, using the boundary condition (3.58) for the last term in (3.67), we get

$$
I I=-\frac{1}{2} \sum_{j=2}^{N-1}\left(\left|\frac{u_{j+1}^{k+1}-u_{j}^{k+1}}{\delta y}\right|^{2}-\sum_{j=2}^{N-1}\left|\frac{u_{j+1}^{k}-u_{j}^{k}}{\delta y}\right|^{2}\right)-\frac{\delta t}{\delta y}\left|\frac{\widetilde{u}_{N}-\widetilde{u}_{N-1}}{\delta y}\right|^{2} .
$$

and the result follows since $I+I I=0$.

Now, we give some numerical illustrations for this boundary condition and we compare the results with the results obtained with the usual Neumann boundary condition $\partial_{y} u=0$ at point $y=1$. The Neumann boundary condition is expressed in terms of the unknown $u_{N}^{k+1}$ by $u_{N}^{k+1}=u_{N-1}^{k+1}$ The initial condition is taken as $u_{0}(y)=e^{-50(y-0.5)^{2}}$, we take $\varepsilon_{1}=1.510^{-2}, \varepsilon_{2}=10^{-3}, \varepsilon_{2}=10^{-4}, N=512$ and $\delta t=\delta x$.

In figures 4,5 and 6 , we see that the solution $u$ propagates in the $y$-positive direction and leaves the simulation box with some reflections of size $\varepsilon$. Moreover the dispersive effects are more important when $\varepsilon$ is greater. Finally in the last plot (figure 7, discrete $L^{2}$-norm in function of time), we see that, when the solution leaves the domain, the energy decays with time which is in accordance with estimate (3.54). We will see that for the full system, since these reflections are small, the behavior of the solutions will be not affected in the interior of the domain by the boundary.

We perform the same computations using Neumann boundary conditions $\partial_{y} u=0$ at the point $y=1$ for $\varepsilon=0.001$. Figure 8 shows that the solution $u$ leaves the domain at the point $y=1$ with more reflections than in the case where we use the boundary condition (3.58). 




Figure 4: Modulus of the solution $u(t)$ of the linear Schrödinger equation (3.50) endowed with the boundary condition (3.53) at time : $t=5,510^{-2}(\mathrm{~A}), t=0.5(\mathrm{~B}), t=0.77(\mathrm{C})$, $t=1.6(\mathrm{D})$ for $\varepsilon=0.015$. The scale of picture $\mathrm{D}$ is not the same than that of $(\mathrm{A})-(\mathrm{B})-(\mathrm{C})$.

\subsubsection{Boundary condition for the wave equation}

We now focus on the wave equation (2.15) describing the evolution of the fluctuation density of ion

$$
\left(\partial_{t}^{2}-v_{s}^{2} \partial_{y}^{2}\right) \delta n=\frac{m_{e}}{4 m_{i}} \frac{k_{0}^{2}}{k_{D e}^{2}} \frac{\omega_{p e}^{2}}{\omega_{0}^{2}} \partial_{y}^{2}|E|^{2}+\frac{m_{e}}{4 m_{i}} v_{s} \partial_{y}^{2}\left(\left|A_{0}\right|^{2}+\left|A_{R}\right|^{2}\right),
$$

and more particularly on the source term (the ponderomotive force). This source term contains three terms that propagate at different velocities. The first term $\partial_{y}^{2}|E|^{2}$ describes the fluctuation due to the propagation of the longitudinal electronic plasma waves. The second and the third terms $\partial_{y}^{2}\left(\left|A_{0}\right|^{2}+\left|A_{R}\right|^{2}\right)$ describe the fluctuation of density due to the propagation of the electromagnetic laser field and the Raman backscattered wave. The key point is that in physical applications, the velocity $v_{s}$ of the acoustic waves is small compared to, the group velocity $v_{0}$ of the laser and the group velocity $v_{R}$ of the Raman field. Moreover the group velocity of electronic plasma waves is also small compared to the electromagnetic waves. Therefore the significant part is

$$
\begin{aligned}
& \partial_{t}^{2} \delta n-v_{s}^{2} \partial_{y}^{2} \delta n=\partial_{y}^{2} f_{0}\left(y-v_{0} t\right)+\partial_{y}^{2} f_{R}\left(y-v_{R} t\right), \quad 0 \leq y \leq 1, \\
& \delta n(0, y)=0, \quad 0 \leq y \leq 1, \\
& \partial_{t} \delta n(0, y)=0, \quad 0 \leq y \leq 1,
\end{aligned}
$$

where $v_{0}>0, v_{R}<0$ and $v_{s}$ is a small positive parameter such that $v_{s} \ll v_{0},\left|v_{R}\right|$. Here, $f_{0}$ and $f_{R}$ are given functions and they refer to the fields $A_{0}$ and $A_{R}$ respectively. The 


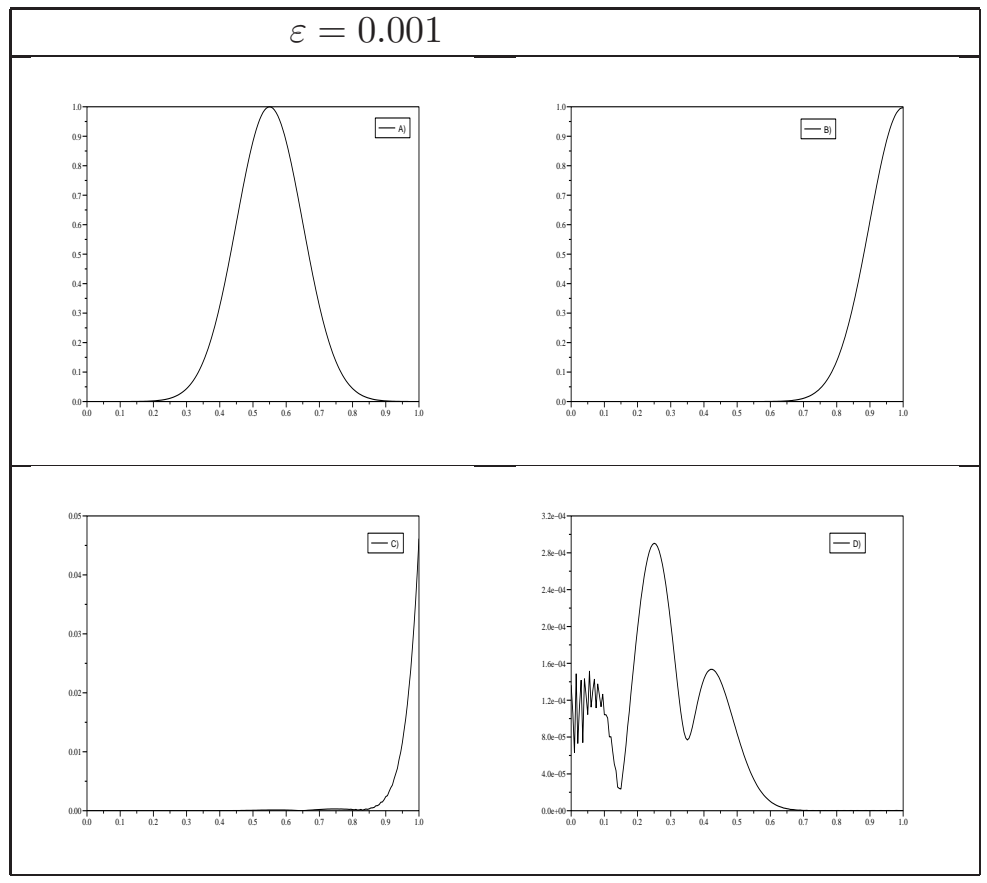

Figure 5: Modulus of the solution $u(t)$ of the linear Schrödinger equation (3.50) endowed with the boundary condition (3.53) at time : $t=5,510^{-2}(\mathrm{~A}), t=0.5(\mathrm{~B}), t=0.77(\mathrm{C})$, $t=1.6(\mathrm{D})$ for $\varepsilon=0.001$. The scales of figures (C),(D) are not the same than that of (A) and $(\mathrm{B})$. 


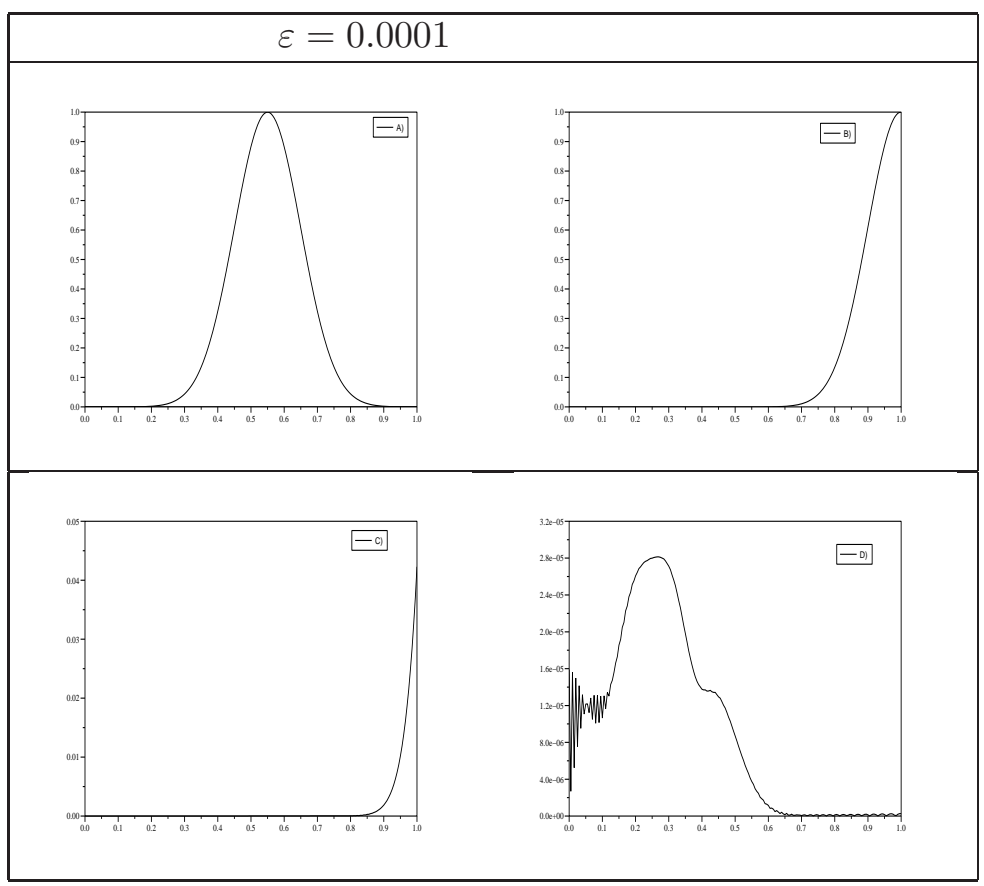

Figure 6: Modulus of the solution $u(t)$ of the linear Schrödinger equation (3.50) endowed with the boundary condition (3.53) at time : $t=5,510^{-2}(\mathrm{~A}), t=0.5(\mathrm{~B}), t=0.77(\mathrm{C})$, $t=1.6(\mathrm{D})$ for $\varepsilon=0.0001$. The scale of figures (C),(D) are not the same than that of (A) and $(\mathrm{B})$.

\begin{tabular}{|c|c|c|c|}
\hline$\varepsilon=0.015$ & $\varepsilon=0.001$ & $\varepsilon=0.0001$ \\
\hline \hline & & & \\
\hline
\end{tabular}

Figure 7: $L^{2}$-norm in function of time for three different values of $\varepsilon$ of the solution $u$ of (3.50) endowed by the boundary condition (3.53). 


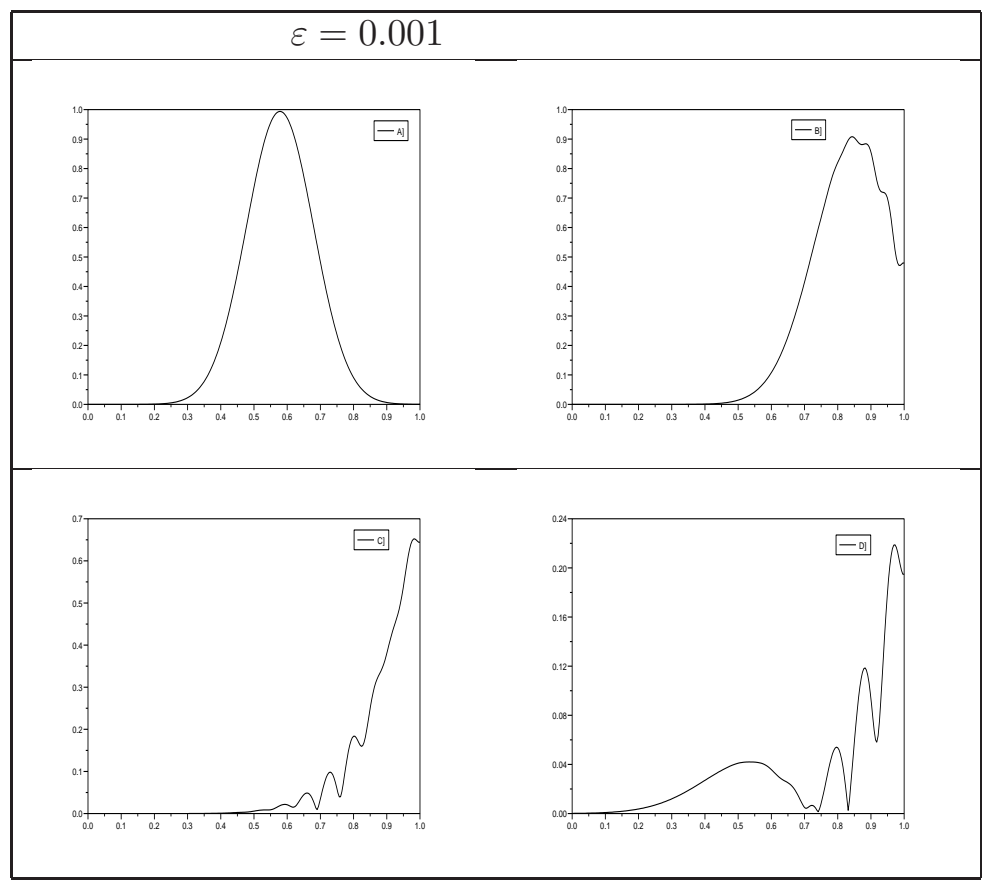

Figure 8: Modulus of the solution $u(t)$ of the linear Schrödinger equation (3.50) at time : $t=5,510^{-2}(\mathrm{~A}), t=0.3(\mathrm{~B}), t=0.8(\mathrm{C}), t=1.2(\mathrm{D})$ for $\varepsilon=0.001$ by using the Neumann boundary condition.

exact solution of (3.70)-(3.72) reads

$$
\delta n(t, y)=\alpha f_{0}\left(y-v_{s} t\right)+\beta f_{0}\left(y+v_{s} t\right)+\gamma f_{0}\left(y-v_{0} t\right)+\delta f_{R}\left(y-v_{R} t\right),
$$

where $\alpha, \beta, \gamma$ and $\delta$ are real constant.

This explicit solution for $\delta n$ shows us that the perturbations of density due to the source terms $\partial_{y}^{2} f_{0, R}\left(y-v_{0, R} t\right)$ propagate more rapidly than $\alpha f_{0}\left(y-v_{s} t\right)+\beta f_{0}\left(y+v_{s} t\right)$. We work on a time scale for the full system for which this perturbation at velocity $v_{s}$ does not have the time to leave the computational domain. It is therefore sufficient to build absorbing boundary conditions for the perturbations which leave the domain at velocity $v_{0}$ respectively $v_{R}$ at point $y=L$ respectively at point $y=0$. To ensure this, the boundary conditions for $\delta n$ consists in our case in the following first order boundary conditions

$$
\begin{gathered}
\partial_{t} \delta n+v_{R} \partial_{y} \delta n=0, \quad y=0, \\
\partial_{t} \delta n+v_{0} \partial_{y} \delta n=0, \quad y=1 .
\end{gathered}
$$

In order to validate this kind of boundary condition, we use the scheme introduced by Glassey [11]. It reads

$$
\frac{\delta n^{k+1}-2 \delta n^{k}+\delta n^{k-1}}{\delta t^{2}}-v_{s}^{2} D_{+} D_{-}\left(\frac{\delta n^{k+1}+\delta n^{k-1}}{2}\right)=D_{+} D_{-}\left(f_{0}^{k}+f_{R}^{k}\right),
$$

where the discret operator $D_{+} D_{-}$is defined by

$$
\left(D_{+} D_{-} \delta n\right)_{i}=\frac{\delta n_{i+1}-2 \delta n_{i}+\delta n_{i-1}}{\delta y^{2}}, i=2, N-1 .
$$


The quantities $\delta n_{1}^{k}, \delta n_{N}^{k}$ are given by the following discretization of the boundary conditions (3.74)-(3.75) at each time step $k$

$$
\begin{gathered}
\delta n_{1}^{k+1}=\left(1+\frac{v_{R} \delta t}{\delta y}\right) \delta n_{1}^{k}-\frac{v_{R} \delta t}{\delta y} \delta n_{2}^{k} \\
\delta n_{N}^{k+1}=\left(1-\frac{v_{0} \delta t}{\delta y}\right) \delta n_{N}^{k}+\frac{v_{0} \delta t}{\delta y} \delta n_{N-1}^{k} .
\end{gathered}
$$

For the numerical illustrations of this problem, we take $v_{s}=0.15, v_{0}=0.9, v_{R}=-0.8$, $f_{0, R}(t, y)=0.02 e^{-\frac{\left(y-\frac{1}{2}-v_{0}, R^{t}\right)^{2}}{2}}, N=1024$ and $\delta t=\delta y \min \left(\frac{1}{v_{0}}, \frac{1}{\left|v_{r}\right|}\right)$. The initial conditions $n_{0}$ and $n_{1}$ are set to zero.
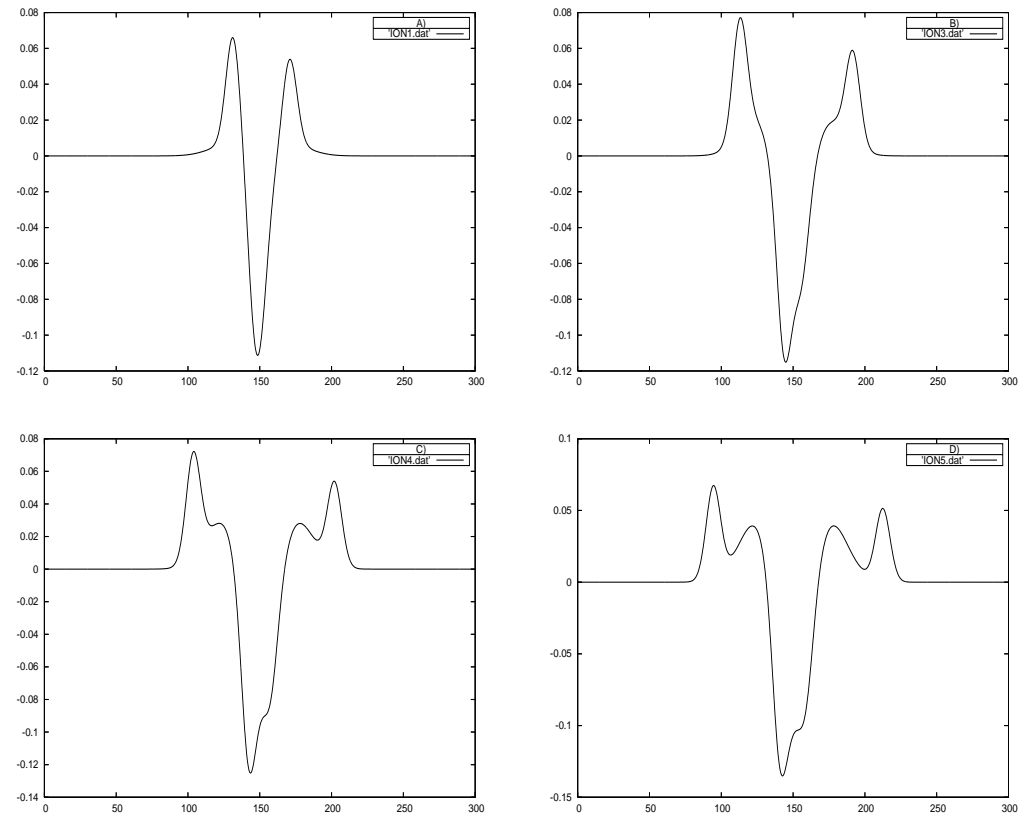

Figure 9: Snapshop of density fluctuation $\delta n$ solution of (3.70)-(3.72) endowed with the boundary conditions (3.74)-(3.75) at time $t=11,6$ for $A), t=35$ for $B), t=47$ for $C$ ), $t=59$ for $D)$.

On figure 9, we can see the evolution of the density fluctuation with time. At the beginning of the simulation, a perturbation due to the force $f_{0}, f_{R}$ causes a sink of density. This perturbation propagates at four velocities which are $v_{R},-v_{s}, v_{s}, v_{0}$. It is obvious that the perturbations propagating at velocity $v_{R}, v_{0}$ go more quickly than the others propagating at velocity $-v_{s}, v_{s}$. The part of the perturbation traveling at velocity $v_{R}\left(v_{0}\right)$ leaves the simulation box at point $y=0(y=1)$, (see figure 10). The perturbations going at velocity $\pm v_{s}$ still propagate. 

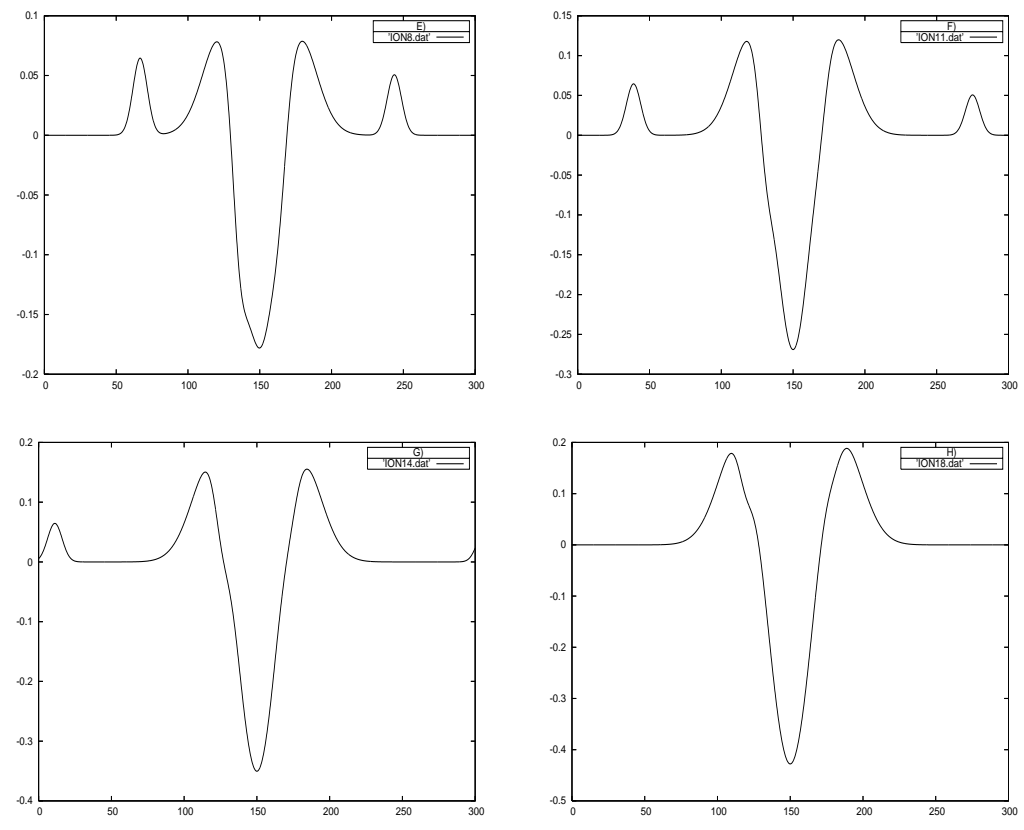

Figure 10: Snapshop of density fluctuation $\delta n$ solution of (3.70)-(3.72) endowed with the boudary conditions (3.74)-(3.75) at time $t=95$ for $E), t=126$ for $F), t=162$ for $G$ ), $t=208$ for $H$ ).

Remark 3.3. At this point, we gave some efficient absorbing boundary conditions for a linear Schrödinger equation and for a wave equation. For the linear Schrödinger equation endowed by the boundary condition (3.50), we found an estimate which shows us that the energy of the solution decrease with time. Unfortunately, we are not able to give such a theoretical result on a nonlinear version of this kind of system. Nevertheless, in the next part, we show that we can apply this kind of boundary conditions on a Zakharov model with a satisfactory numerical accuracy.

\subsubsection{The boundary conditions for the Zakharov system.}

We consider the following Zakharov model

$$
\begin{aligned}
& i\left(\partial_{t} u+v \partial_{y} u\right)+\varepsilon \partial_{y}^{2} u=n u, 0<y<1, \\
& \partial_{t}^{2} \delta n-v_{s}^{2} \partial_{y}^{2} \delta n=\partial_{y}^{2}\left(|u|^{2}\right), 0<y<1, \\
& u(0, y)=u_{0}(y) \\
& u(t, 0)=0 \\
& \partial_{t} u+v \partial_{y} u=0, y=1 \\
& \delta n(0, y)=0, \partial_{t} \delta n(0, y)=0 \\
& \partial_{t} \delta n+v \partial_{y} \delta n=0, y=1 .
\end{aligned}
$$

Even if we are not able to justify rigorously our set of boundary conditions for the full Zakharov system (3.80)-(3.86), the numerically accuracy is satisfactory. Indeed, in Fig 11, we have plotted the modulus of electric field $u$ and the variation of density $\delta n$ with respect 
to time. As seen on the snapshots, no visible reflexions can be seen on any of the curves. For the simulation described in Fig 11, we have taken $\delta t=\delta y=0.01$. Of courses, these results has to be confirmed on the complete system
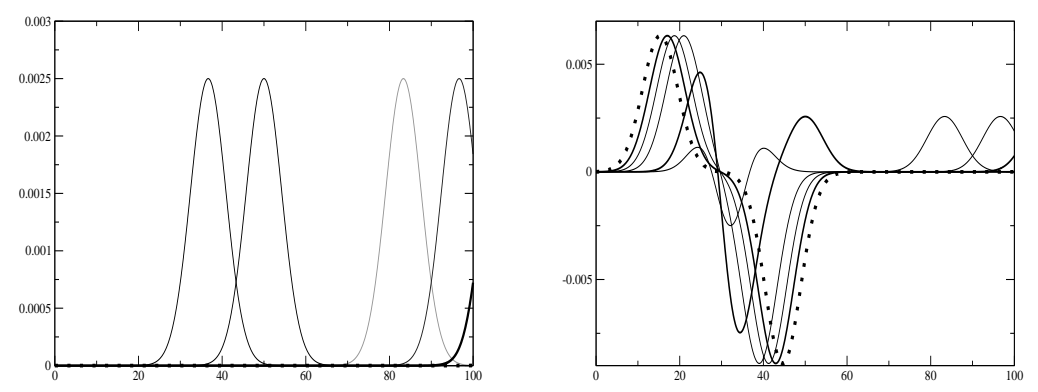

Figure 11: $|u|(y)$ (left )and $\delta n(y)$ (right) solution of $(3.80),(3.86)$ at different time for $\varepsilon=5.10-3$.

\subsubsection{Boundary condition for the full system}

We now adapt our set of boundary conditions to

$$
\begin{aligned}
& \partial_{t} A_{0}+v_{0} \partial_{y} A_{0}=0, \quad y=L, \\
& \partial_{t} A_{R}+v_{R} \partial_{y} A_{R}=0, \quad y=0, \\
& \partial_{t} \delta n+v_{0} \partial_{y} \delta n=0, \quad y=L, \\
& \partial_{t} \delta n+v_{R} \partial_{y} \delta n=0, \quad y=0 .
\end{aligned}
$$

Concerning the plasma waves, since the group velocity of the electronic plasma waves is small with respect to $v_{0}$ and $v_{R}$ (see [5],[6]), we use periodic boundary condition for $E$, that is $E(0)=E(L)$. These boundary conditions are discretized as we described previously using a spectral method.

The detailed numerical results are given in the next section. 


\section{Numerical results}

\subsection{Basic simulations}

In this part, we use the dimensionless quasi-linear type Zakharov model. We use the following set of values of parameters that are representative of the physics that is involved.

- the velocity of light is $c=3.10^{8} \mathrm{~ms}^{-1}$,

- the thermal velocity of electrons is taken to be equal to $v_{t h e}=0.1 c$,

- the mass ratio is taken to be $\frac{m_{e}}{m_{i}}=\frac{1}{2000}$,

- the sound velocity is $c_{s}=\sqrt{\frac{m_{e}}{m_{i}}} v_{t h e}$,

- the plasma frequency $\omega_{p e}=3.10^{15} s^{-1}$,

- the wave number of the laser field $k_{0}=3.10^{6}$,

- the Debye's length $\lambda_{D e}=\frac{v_{t h e}}{\omega_{p e}}$.

With this parameters, we compute the frequency $\omega_{0}$ thanks to the dispersion relation

$$
\omega_{0}=\sqrt{\omega_{p e}^{2}+k_{0}^{2} c^{2}}
$$

and search for $k_{R}, k_{1}, \omega_{R}$ and $\omega_{1 d}$ with the following matching conditions for the three waves resonance condition

$$
k_{0}=k_{R}+k_{1}, \omega_{0}=\omega_{R}+\omega_{p e}+\omega_{1 d}
$$

where

$$
\omega_{R}=\sqrt{\omega_{p e}^{2}+k_{R}^{2} c^{2}}, \omega_{1 d}=\frac{2}{\delta t} \arctan \left(\alpha \delta t \frac{1-\cos \left(k_{1} \delta y\right)}{\delta y^{2}}\right)
$$

with a dichotomy process and where $\alpha=\frac{3 k_{0}^{2}}{2 k_{D e}^{2}}$. We compute the system on the spatial domain $[0, L y]$ with $L k_{0}=250$.

For the initial conditions, we consider an gaussian initial data for $A_{0}$ of the form

$$
A_{0}(0, x)=0.3 e^{-0.01(x-40)^{2}} .
$$

Since we deal with simulated Raman instability, we have to begin with a small perturbation on $A_{R}$ and we take $A_{R}(0, x)=0.005 A_{0}(0, x)$. Furthermore, $E, \delta n$ and $\partial_{t} \delta n$ are taken equal to 0 at time $t=0$, (we considerer that the plasma is at equilibrium when $t=0$ ). The initial electron distribution function is assumed to be a Maxwellian :

$$
F_{e}(0, v)=\frac{1}{\sqrt{2 \pi}} e^{-\frac{v^{2}}{2}}
$$

which gives the following initial condition for the Landau damping rate

$$
\widehat{\nu}(0, \xi)=\sqrt{\frac{\pi}{8}} \frac{k_{D e}^{3}}{k_{0}^{3}} \frac{1}{|\xi|^{3}} e^{-\frac{k_{D e}^{2}}{2 k_{0}^{2} \xi^{2}}} .
$$


The number of discretization points in space is $N_{y}=2048$ and we choose $\delta t=\delta y \min \left(\frac{1}{v_{0}}, \frac{1}{\left|v_{r}\right|}\right)$. We perform the simulation on the time interval $\left[0, T_{\max }\right]$ with $T_{\max }=100$.

At the beginning of simulation, the laser field and the stimulated Raman field interact in order to create an electronic plasma waves. These three fields combine in order to create a perturbation on the low-frequency density $\delta n$. The Laser field propagates on the positive $y$-direction and create on its way Raman backscattered wave which propagate on the $y$ negative direction. Concerning the evolution of the spatial profile of the electronic plasma wave created by the three wave resonance, we can see that its amplitude grows with time and therefore we confirm that its velocity is small with respect to the velocity of $A_{R}$ and $A_{0}$ as we can see in its dispersion relation.

The figure 4.1 shows us that the energy exchanges between the different fields. In a first stage, there is a transfer of energy from the laser field to the Raman field and to the electronic plasma waves until an amplification threshold is reached. This stage occurs before time $\omega_{0} t=15$.

In a second stage, the evolution starts to be non-linear and the Landau damping acts. A new plasma waves is created and a transfer of energy between this plasma wave and the electrons take place. This lead to a creation of hot electron tail as we can see on the figure 4.1. The saturation level of the Raman field starts to decrease. 

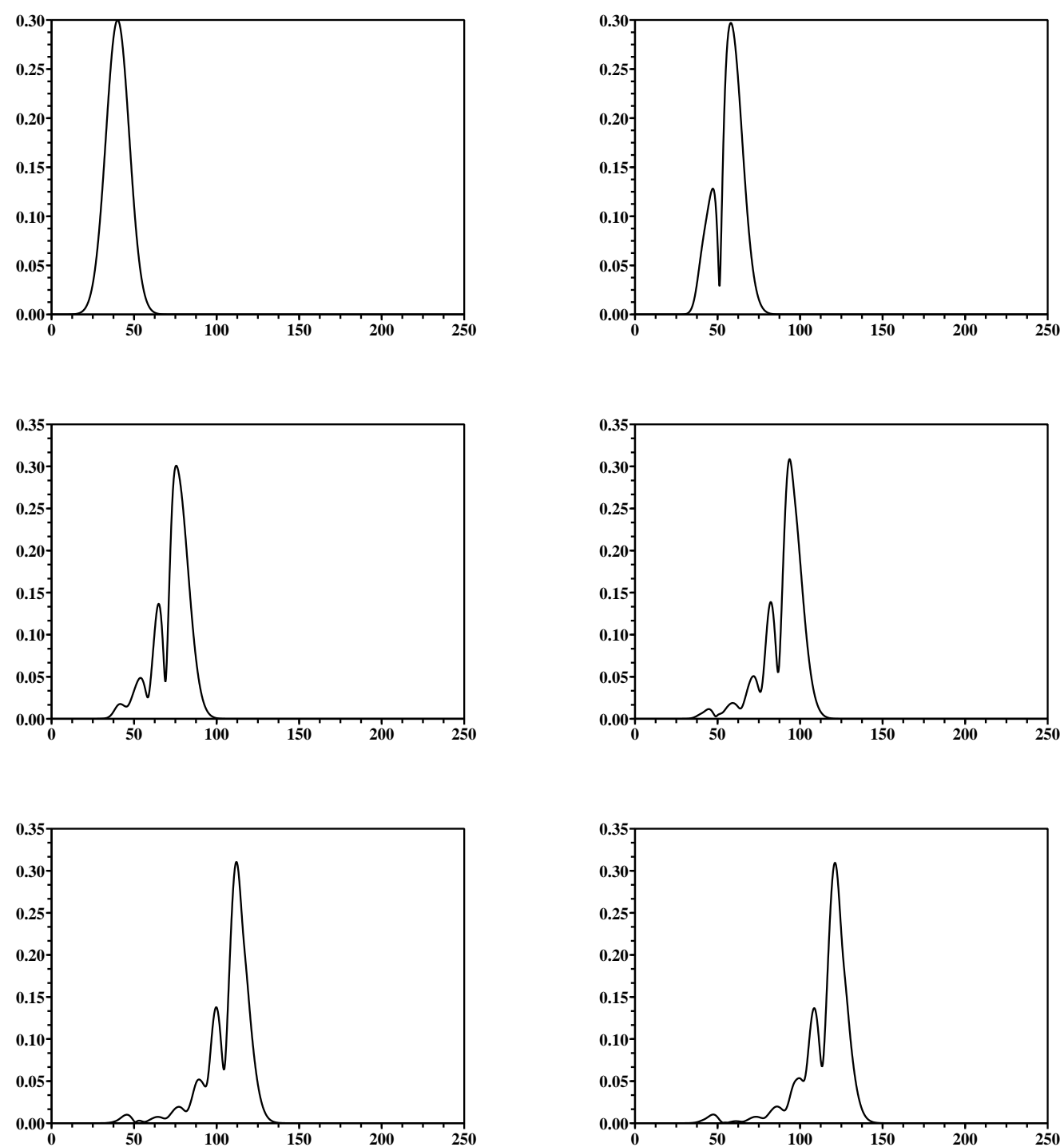

Figure 12: Snapshots of the modulus of the laser field at time $t=10 n$ for $n=1,3,5,7,9,10$ in the resonant case. 

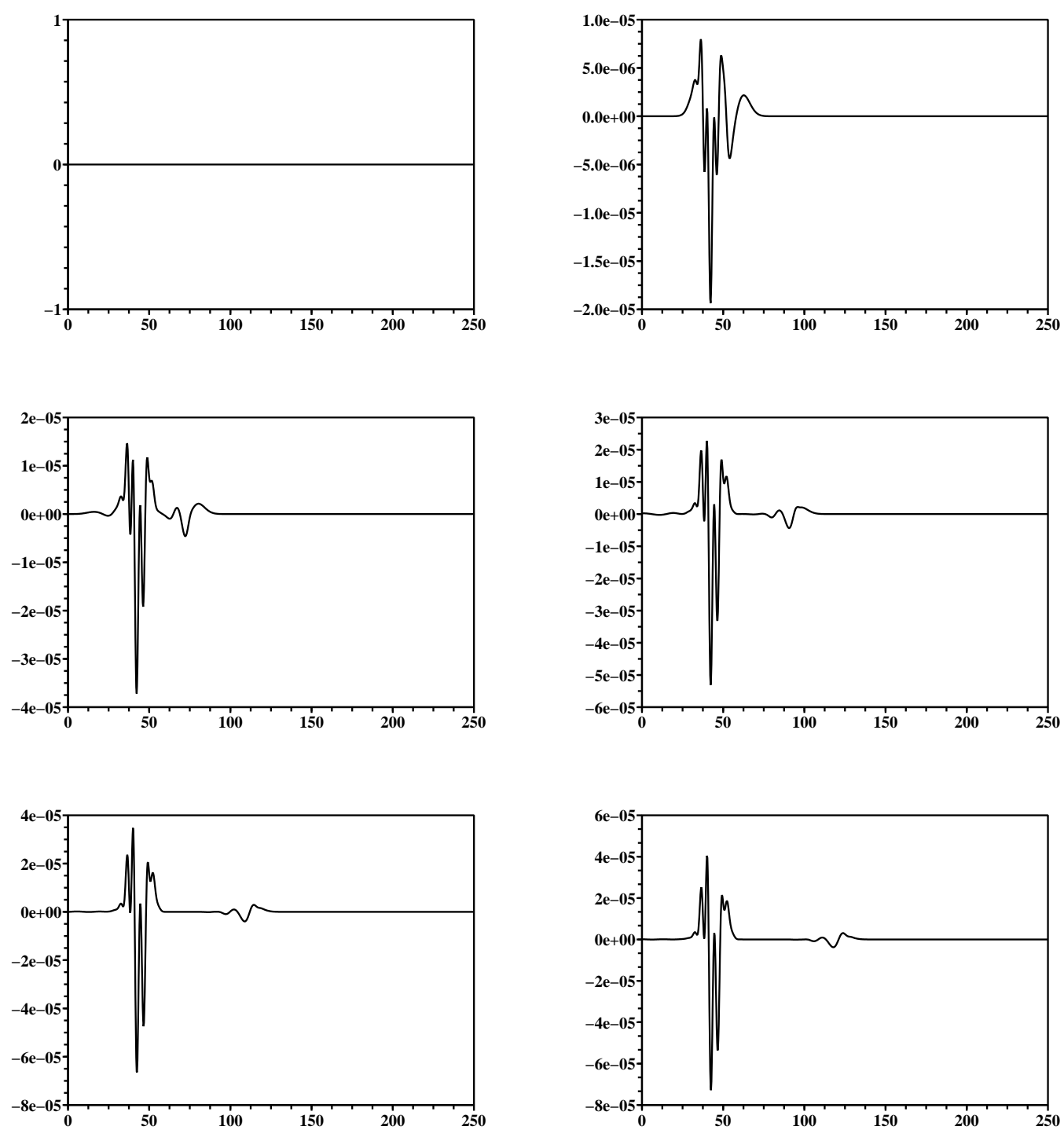

Figure 13: Snapshots of the density fluctuation at time $t=10 n$ for $n=1,3,5,7,9,10$ in the resonant case. 

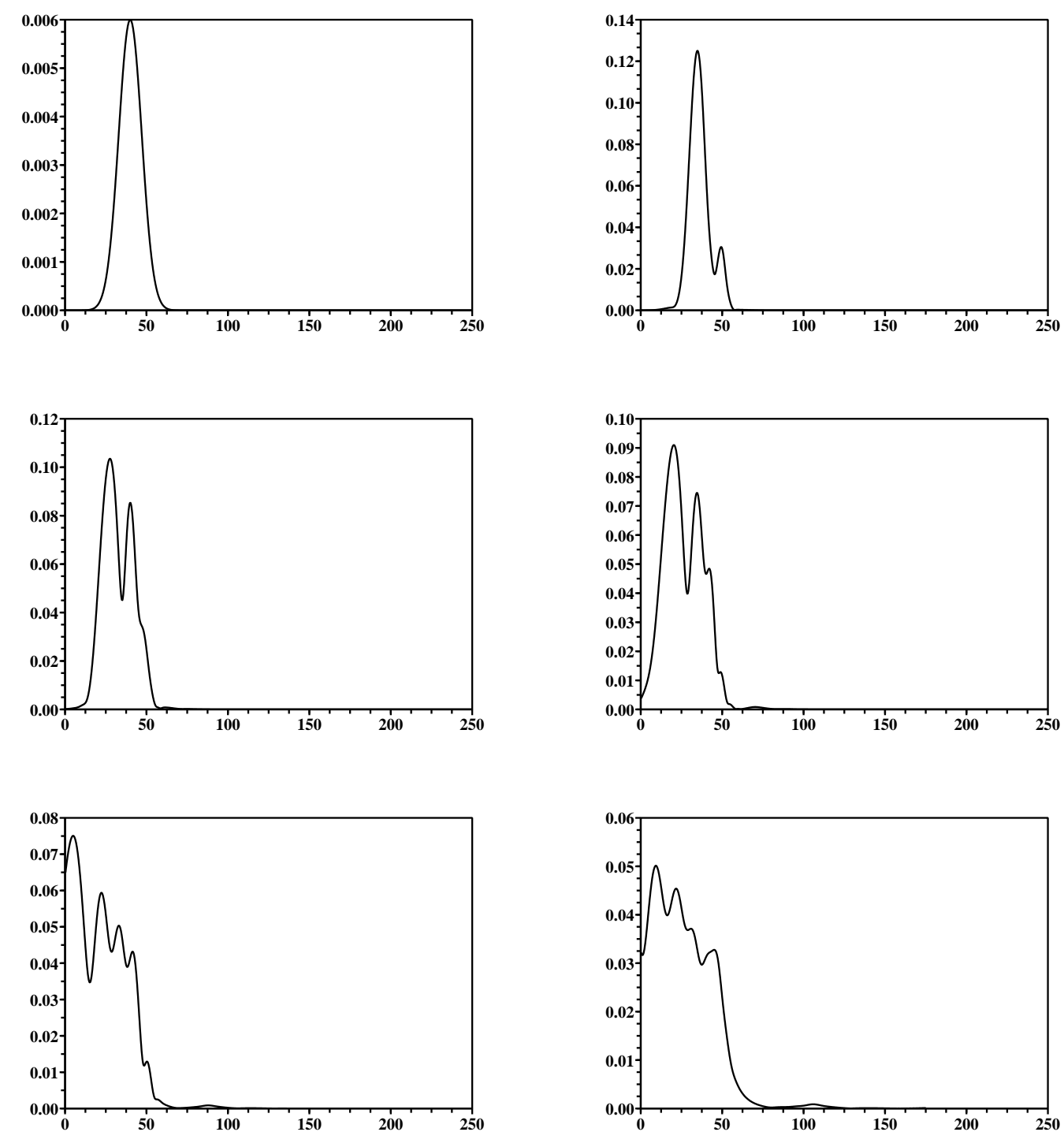

Figure 14: Snapshots of the modulus of the Raman component at time $t=10 \mathrm{n}$ for $n=1,3,4,5,7,10$ in the resonant case. 

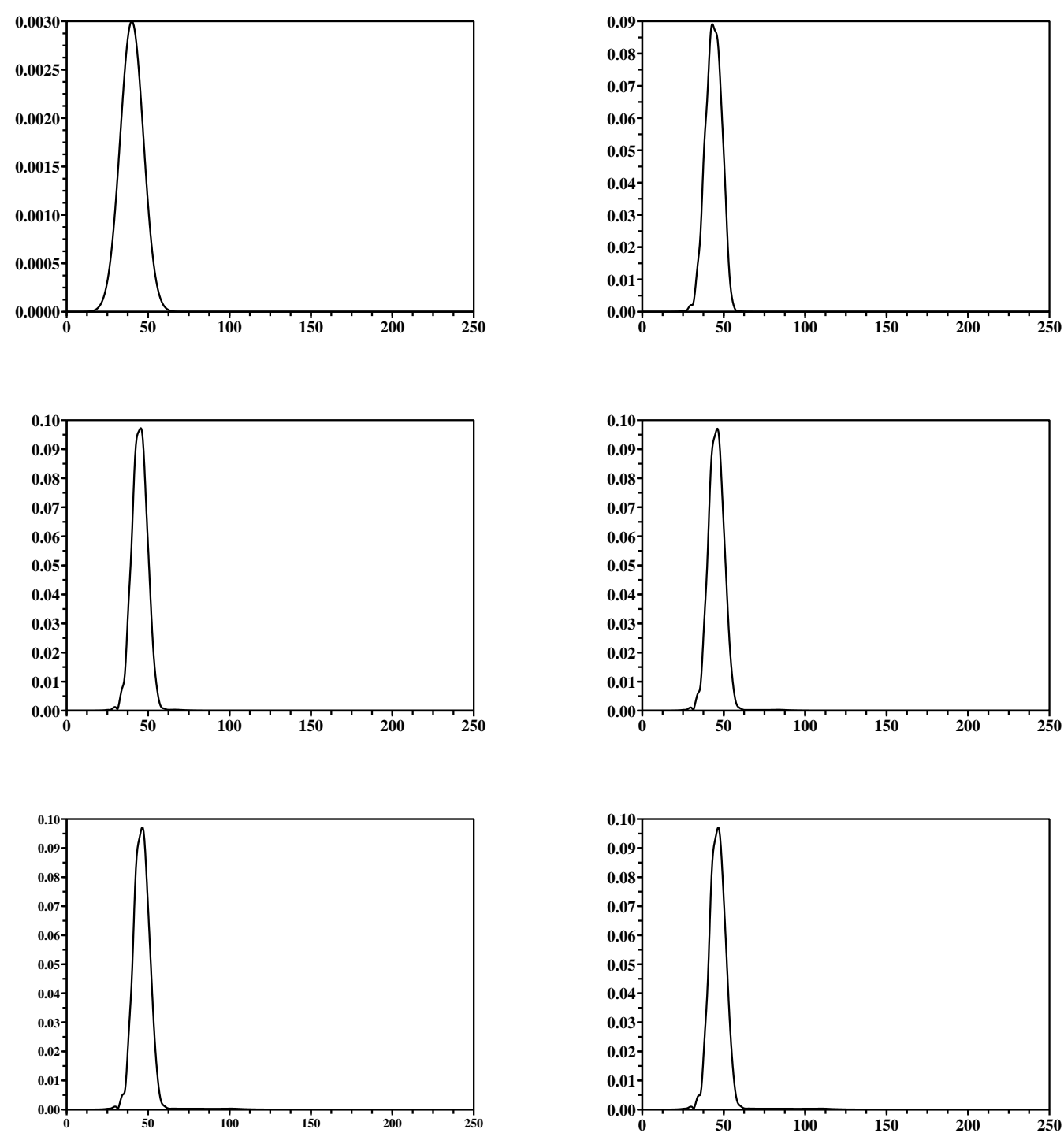

Figure 15: Snapshots of the modulus of the electronic plasma waves at time $t=10 \mathrm{n}$ for $n=1,3,5,7,9,10$ in the resonant case. 


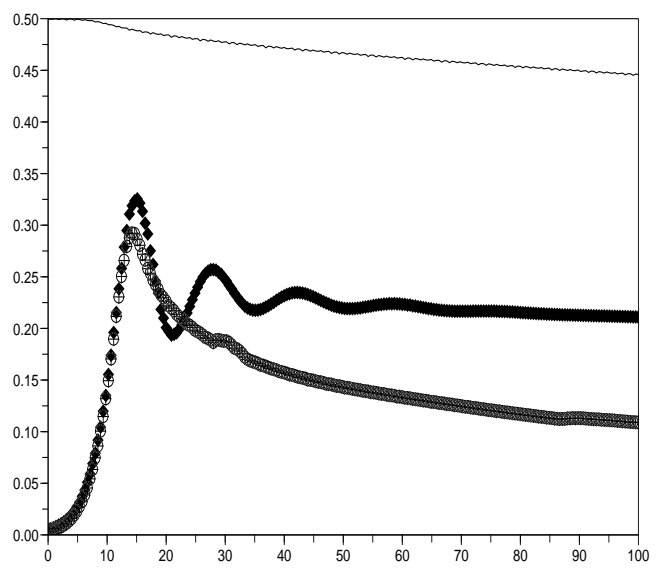

Figure 16: $l^{2}$ discret norm in function of time for $A_{C}$ corresponding with the solid line, $A_{R}$ corresponding with the circle points and $E$ corresponding with the square line.

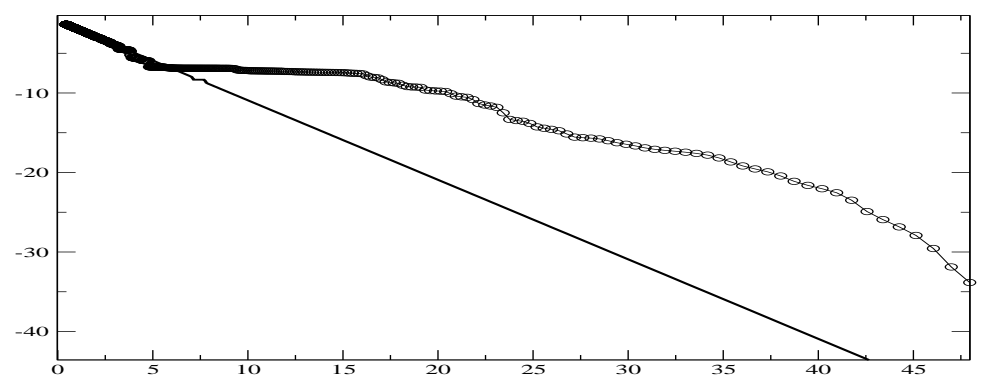

Figure 17: The log of the spatially averaged electron distribution function as a function of the kinetic energy $\frac{v^{2}}{2}$ at $t=5$ (solid line) and at the end of simulation (circle point).

\subsection{Influence of the boundary conditions}

It is clear that for the Schrödinger equation or even for the Zakharov system, some boundary conditions like Neumann ones give rise to reflexions that change deeply the result of the computation. It is not clear that this still the case for the complete system. The aim of this part is to quantify this effect on (2.12)-(2.17). We will perform two different tests. In the first one, we put Neumann boundary conditions for $A_{C}$ and $A_{R}$ and we keep the boundary conditions (3.74)-(3.75) for the density fluctuation of density $\delta n$. In the second one, we use Neumann boundary condition for the density fluctuation and we keep the boundary conditions (3.88)-(3.89) for $A_{C}$ and $A_{R}$. 


\subsubsection{Neumann boundary conditions for $A_{C}$ and $A_{R}$}

The Neumann boundary conditions for $A_{C}$ and $A_{R}$ read

$$
\begin{gathered}
\partial_{y} A_{R}=0, y=0, \\
\partial_{y} A_{C}=0, y=L
\end{gathered}
$$

which give at each time step $k$

$$
\begin{gathered}
A_{R_{1}}^{k}=A_{R_{2}}^{k}, \\
A_{C_{N}}^{k}=A_{C_{N-1}}^{k} .
\end{gathered}
$$

The boundary condition for $\delta n$ is given by

$$
\begin{gathered}
\delta n_{1}^{k+1}=\left(1+\frac{v_{R} \delta t}{\delta y}\right) \delta n_{1}^{k}-\frac{v_{R} \delta t}{\delta y} \delta n_{2}^{k} \\
\delta n_{N}^{k+1}=\left(1-\frac{v_{0} \delta t}{\delta y}\right) \delta n_{N}^{k}+\frac{v_{0} \delta t}{\delta y} \delta n_{N-1}^{k} .
\end{gathered}
$$

We use the same parameters than the first simulation.
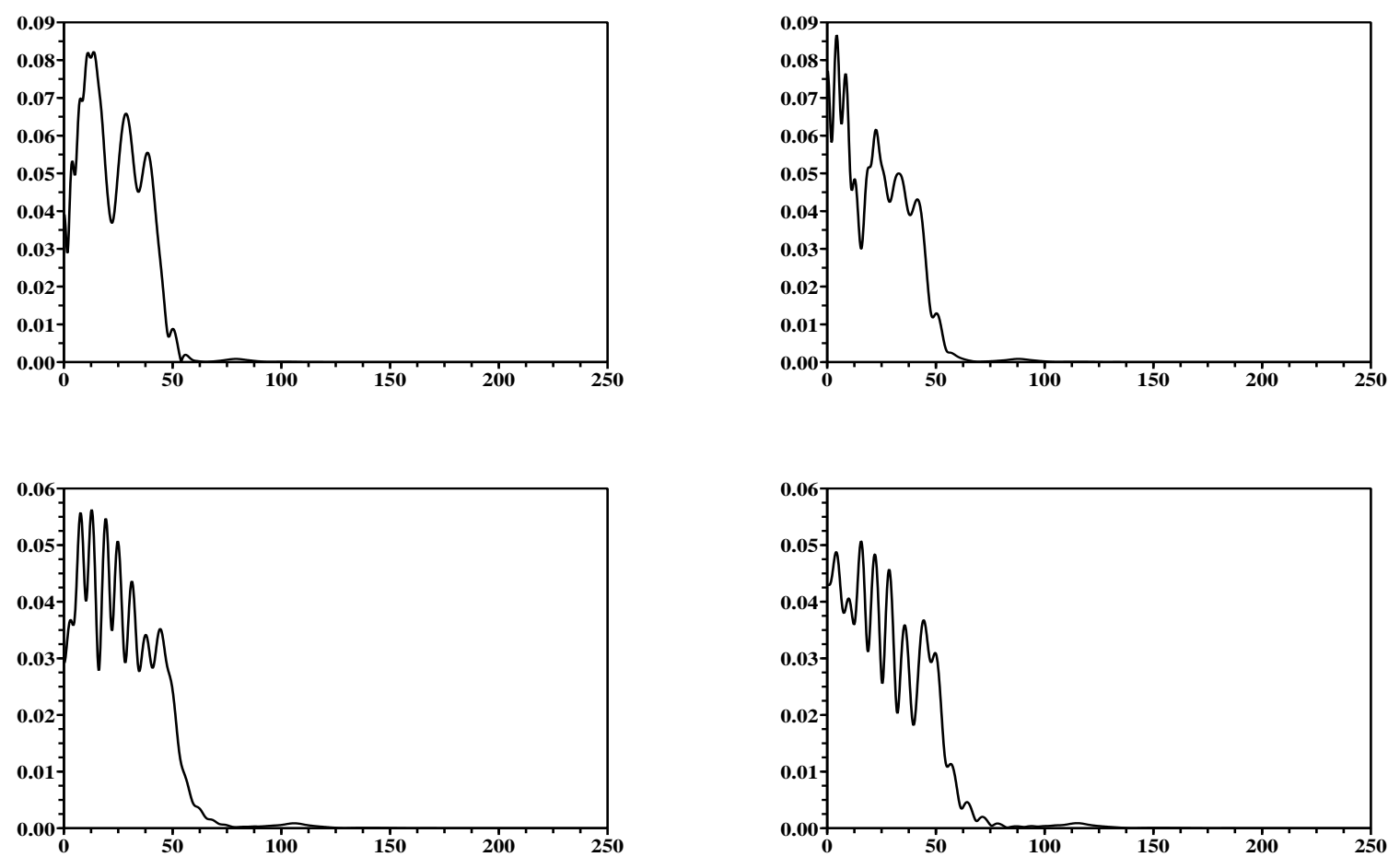

Figure 18: The modulus of the Raman component in space at time (from left to right) at time $t=10 n$ for $n=6,7,9,10$ by using the Neumann boundary conditions on $A_{0}$ and $A_{R}$. 


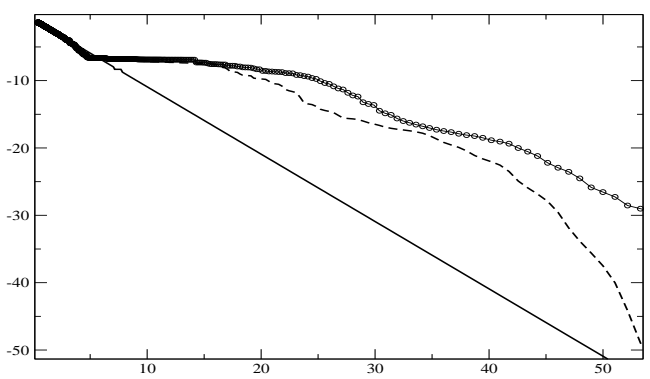

Figure 19: The log of the spatially averaged electron distribution function against kinetic energy $\frac{v^{2}}{2}$ at initial time (solid line) and at the end of simulation : the circle point corresponds to the Neumann boundary conditions on $A_{0}$ and $A_{R}$ and the dashed line corresponds to the boundary conditions (3.80)-(3.86).

As we can see on the left plot of figure 18, when the Raman field leaves the domain at time $t=70$, some reflexions appear and this is clearly caused by the fact that we use Neumann boundary conditions for $A_{R}$ at point $y=0$.

These reflexions are propagated in the $y$ positive direction and modify the electronic plasma waves (and also the electronic plasma waves). So these reflexions modify the electron distribution function as we can see on the figure 19. In this case, more electrons are accelerated.

\subsubsection{Neumann boundary conditions for $\delta n$}

We investigate the symmetric case of previous section. We keep the boundary conditions (3.88)-(3.89) for $A_{0}$ and $A_{R}$ and we put Neumann boundary condition for the density's fluctuation $\delta n$. In figure 20, we can see reflexions on the density fluctuation. This occurs exactly when the Raman field leaves the spatial domain at time $t=60-70$. Note that the reflexions are more important in this case than the previous case. In figure 21, as in the previous case, more electrons are accelerated. 

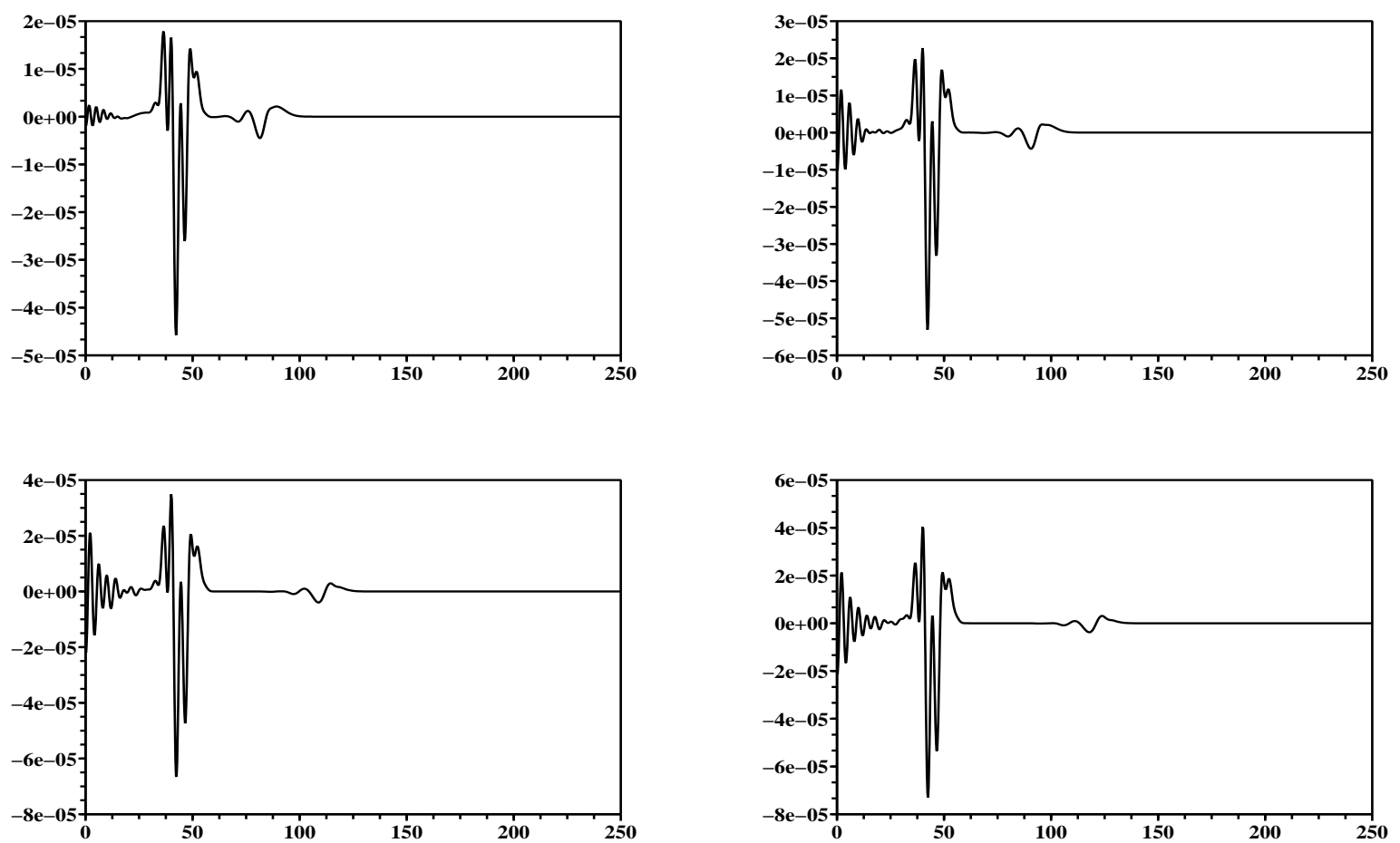

Figure 20: The density fluctuation in space at time (from left to right) at time $t=10 n$ for $n=6,7,9,10$ by using the Neumann boundary condition on $\delta n$.

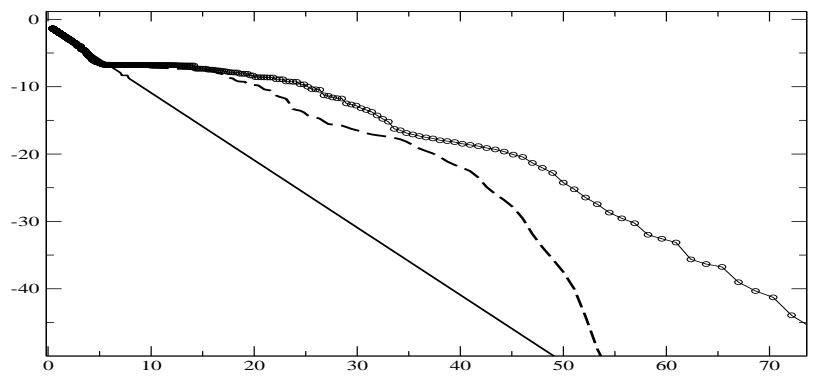

Figure 21: The log of the spatially averaged electron distribution function against kinetic energy $\frac{v^{2}}{2}$ at initial time (solid line) and at the end of simulation : the circle point corresponds to the Neumann boundary condition on $\delta n$ and the dashed line corresponds to the boundary conditions (3.80)-(3.86). 


\subsection{Influence of the dispersion relation}

In this part, we emphasize the fact that it is important to use the numerical dispersion relation (3.43). For this, we compare the growth of the maximum amplitude of the electronic plasma waves in two case. The first one concerns the numerical dispersion relation and the second one deals with the theoretical dispersion relation.

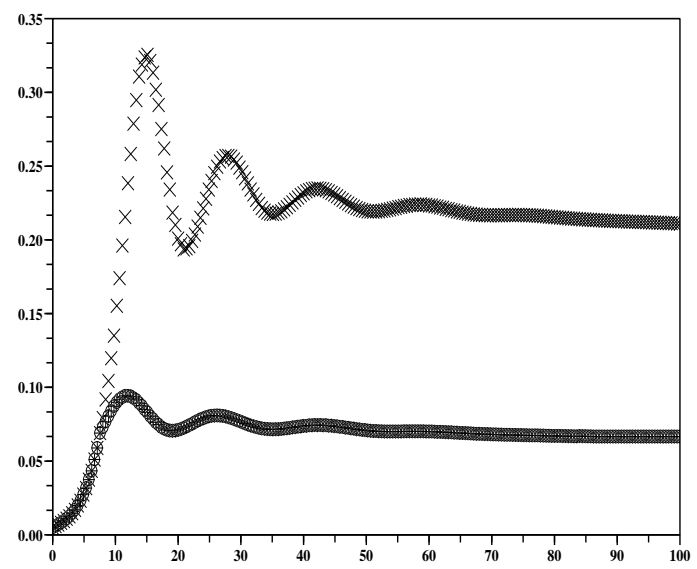

Figure 22: The maximum of amplitude of $|E|$ in function of time. The dotted-line correspond with the numerical dispersion relation, and the circle line correspond with the theoretical dispersion relation.

As in the case of the linear Schrödinger equation, figure 22 show us that when we use the theoretical relation dispersion, we underestimate the growth of the amplitude of the electronic plasma waves and it has an influence on the number of accelerated electrons as we can see in figure 23. In this plot, we can see that when we use the numerical dispersion relation then more electron are accelerated which is an accordance with the fact that the maximum of amplitude of $E$ is more higher than in a non-resonant case. 


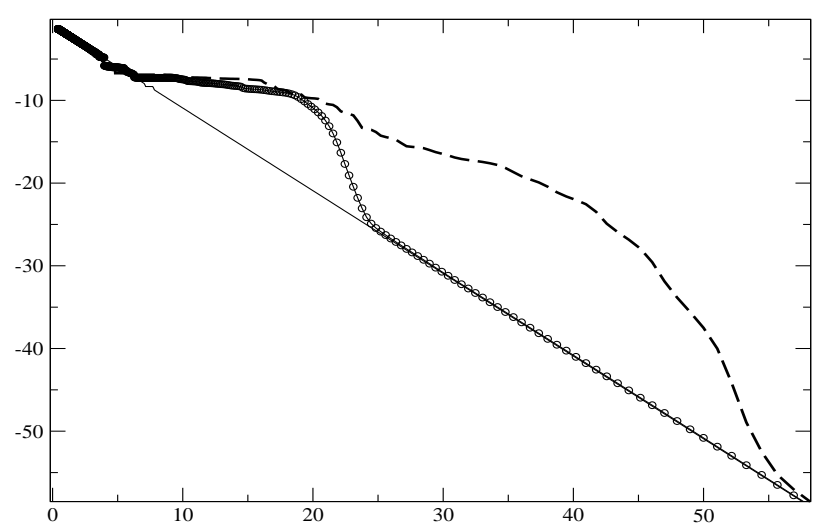

Figure 23: The spatially averaged electron distribution function against kinetic energy, for the numerical dispersion relation (dashed line) and for the theoretical dispersion relation (circle line).

\subsection{Influence of the Landau damping process}

In order to show how the Landau damping process works on the saturation of the Raman amplification, we compare the results with the case where the Landau damping rate is close to zero. We focus on the evolution in time of the discret $l^{2}$ norms $|E|_{2}^{2}$. In the case where the Landau damping process can be neglected, we remark (in figure 24 ) that the growth of the electronic plasma amplitude and the Raman amplification is the same than the case where we take into account the Landau process. This means that the Landau damping process plays no role in this amplification process.

After this first step, we observe that during the saturation of the electronic plasma wave (non linear process), the energy of plasma waves is greater.

This corresponds with the fact, in this case, electrons play no role and plasma waves can not give some energy. It follows ( it depends on the parameter $\frac{k 1}{k_{D e}}$, see [3] for more details) that the level of the Raman saturation decreases. 


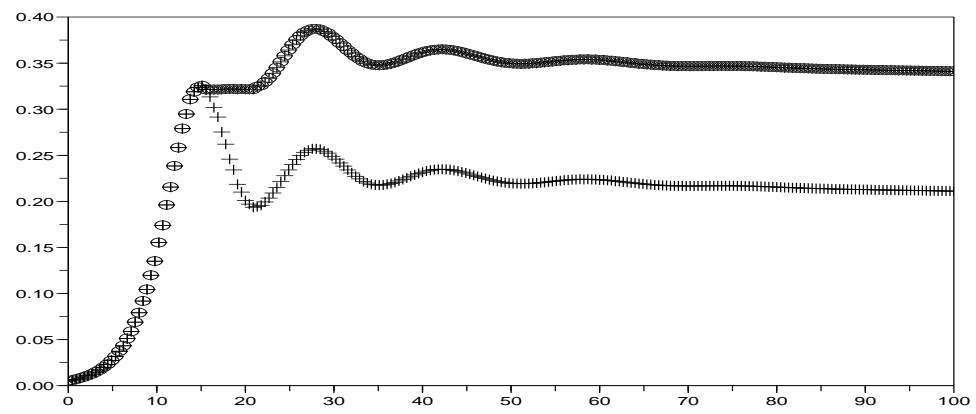

Figure 24: The $l^{2}$ norm of $|E|$ in function of time without Landau damping (circle line) and with Landau damping (dotted line) by using the numerical dispersion relation.

\subsection{Comparison with a first study}

In this part, we want to compare our results with those obtained in [2]. To this aim, in order to recover the results of [2], we fixed the potential $A_{0}, A_{R}$ as being localized Maxwellian functions $A_{0, R}(x) \approx e^{-a x^{2}}$ where $a$ is a constant and we only solve equations (2.14)-(2.17) with the following initial conditions $E(0, y)=0, \delta n(0, y)=0, \partial_{t} \delta n(0, y)=$ $0, H(0, \xi)=\sqrt{\frac{\pi}{8}} \frac{k_{D e}^{3}}{k_{0}^{3}|\xi|^{3}} e^{-\frac{1}{2 \xi^{2}}}$.

The energy is brought into this system by the source term of the Schrödinger equation (2.14) $\partial_{y}\left(A_{0} A_{R}^{*} e^{i\left(k_{1} y-\omega_{1} t\right)}\right)$ and since we choose $a$ such that $\sqrt{a} \ll k_{1}$, the more important part of this source term is $k_{1} A_{0} A_{R}^{*} e^{i\left(k_{1} y-\omega_{1} t\right)}$. We take the same physical parameters that the first basic simulation and we want to compare the energy gain of the electrons in each case. We fixed the amplitude of $A_{0}$ and $A_{R}$ at the values found at the end of the basic simulation.

Since at time $t=0$, the electron distribution function is a Maxwellian, the electron energy is $\int v^{2} F_{e}(0, v) d v=1$. At the beginning of the simulation, Landau damping plays no role in both cases and this corresponds to the first stage of the simulation (figure 25). For the full system (2.12)-(2.17), the second stage starts at time $t=20$ when a significant part of energy is given to electrons. This corresponds to the decay of the amplitude of the electronic plasma waves due to the interaction between the wave-wave and wave-particle processes (see 4.1).

The saturation of the electron energy (figure 25) occurs almost simultaneously with the wave energy saturation (see 4.1).

Concerning the case where we fixed the potential $A_{0}, A_{R}$, the behavior in time of the electron energy is about the same than for the full system. Nevertheless, since the amplitude of the source term $k_{1} A_{0} A_{R}^{*} e^{i\left(k_{1} y-\omega_{1} t\right)}$ of the Schrödinger equation (2.14) does not not evolves with time, the amplitude of the electronic plasma wave created in this case is more important. So the electron energy increases earlier and saturates with a higher level than for the full system.

This means that the system used in [2] overestimates the exchanged energy between the electronic plasma waves and the electrons. 


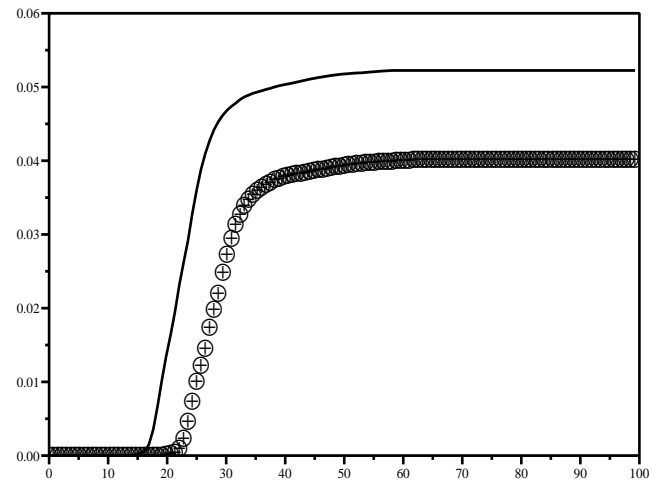

Figure 25: Time evolution the electron energy $\frac{1}{2} \int v^{2} F_{e}(t, v) d v-\frac{1}{2}$ for the full system (circle line) and for fixed $A_{0}, A_{R}$ (solid line).

\section{References}

[1] X. Antoine, C. Besse. Mathematical and numerical analysis of nonlinear artificial boundary conditions for the one-dimensional Schrödinger equation. To appear in SIAM J. Numer. Anal.

[2] R. Belaouar, T. Colin, G. Gallice, C. Galusinski. Theorical and numerical study of a quasilinear Zakharov system describing Landau damping. To appear in M2AN.

[3] R. Belaouar, T. Colin, G. Gallice, V. Tikhonchuk. Quasilinear electron acceleration in a driven plasma wave. Preprint.

[4] C. Besse. Schéma de relaxation pour l'équation de Schrödinger non linéaire et les systèmes de Davey et Stewartson. C.R. Acad. Sci. Paris. Sér. I Math., Vol. 326, (1998), 1427-1432.

[5] M. Colin, T. Colin. On a quasilinear Zakharov System describing laser-plasma interactions. Differential and Integral Equations, 17 (2004), no. 3-4, 297-330.

[6] M. Colin, T. Colin. A numerical model for the Raman amplification for laser-plasma interaction. Journal of computational and Applied Mathematics, 193 (2006), no. 2, $535-562$.

[7] T. Colin, G. Métivier. Instabilities in Zakharov equations for laser propagation in a plasma, in Phase space analysis of PDEs, A. Bove, F. Colombini, D. Del Santo Ed., Progress in Nonlinear Differential equations and their Applications 69, Birkhäuser, 2006.

[8] J-L. Delcroix and A. Bers. "Physique des plasmas 1, 2". Inter Editions-Editions du CNRS, (1994). 
[9] P. Degond. Spectral theory of the linearized Vlasov-Poisson equation. Trans. Amer. Math. Soc. 294 (1986), no. 2, 435-453.

[10] L. Di Menza Transparent and artificial boundary conditions for the linear Schrödinger equation. Numer. Funct. Anal. Optimiz., vol. 18, no. 7-8, pp. 759-776 (1998).

[11] R. T. Glassey Convergence of an energy-preserving scheme for the Zakharov equations in one space dimension, Math. Comp. 58 (1992), no. 197, 83-102.

[12] R. T. Glassey, J. Schaeffer. On time decay rates in Landau damping. Comm. Partial Differential Equations 20 (1995), no. 3-4, 647-676.

[13] D.E Knuth, The art of computer programming. Addison-Wesley Publishing Company, Menlo Park, California, (1969).

[14] G.L. Payne, D.R. Nicholson and R.M. Downie, Numerical Solution of the Zakharov Equations, J. Compt. Phys., 50, 482-498 (1983).

[15] G. Riazuelo. Etude théorique et numérique de l'influence du lissage optique sur la filamentation des faisceaux lasers dans les plasmas sous-critiques de fusion inertielle. Thèse de l'Université Paris XI.

[16] D.A. Russel, D.F. Dubois and H.A. Rose. Nonlinear saturation of simulated Raman scattering in laser hot spots. Physics of Plasmas, Vol. 6 (4), (1999), 1294-1317.

[17] K. Y. Sanbomatsu , Competition between Langmuir wave-wave and wave-particule interactions, Thesis of University of Colorado, Department of Astrophysical, 1997.

[18] K. Y. Sanbomatsu et. al., The effect of kinetic processes on Langmuir turbulence, Phys. Plasmas 7, 1723 (2000).

[19] K. Y. Sanbomatsu et. al., Quantitative comparison of reduced-description particle-incell and quasilinear-Zakharov models for parametrically excited Langmuir turbulence, Phys. Plasmas 7, 2824 (2000).

[20] S. Schochet and M. Weinstein. The nonlinear Schrödinger limit of the Zakharov equations governing Langmuir turbulence. Comm. Math. Phys., Vol. 106, (1986), 569-580.

[21] C. Sulem and P-L. Sulem. "The nonlinear Schrödinger Equation. Self-Focusing and Wave Collapse." Applied Mathematical Sciences 139, Springer, (1999).

[22] V.E. Zakharov, S.L. Musher and A.M. Rubenchik. Hamiltonian approach to the description of nonlinear plasma phenomena. Phys. Reports, Vol. 129, (1985), 285-366. 